\title{
Error bounds for a difference scheme approximating viscosity solutions of mean curvature flow
}

\author{
KLAUS DECKELNick \\ Centre for Mathematical Analysis and its Applications, School of Mathematical Sciences, \\ University of Sussex, Falmer, Brighton BN1 9QH, UK \\ [Received 18 December 1998 and in revised form 13 August 1999]
}

\begin{abstract}
We analyse a finite difference scheme for the approximation of level set solutions to mean curvature flow. The scheme which was proposed by Crandall \& Lions (Numer. Math. 75, (1996) 17-41) is a monotone and consistent discretization of a regularized version of the underlying problem. We derive an $L^{\infty}$-error bound between the numerical solution and the viscosity solution to the level set equation provided that the space and time step sizes are appropriately related to the regularization parameter.
\end{abstract}

\section{Introduction}

The aim of this paper is to prove an error estimate for a difference scheme approximating mean curvature motion in its level set formulation. This approach can be described as follows: let $\Gamma_{0} \subset$ $\mathbb{R}^{n}, n \geqslant 2$ be a given initial hypersurface and choose a continuous function $u_{0}: \mathbb{R}^{n} \rightarrow \mathbb{R}$ such that $\Gamma_{0}$ corresponds to the zero level set of $u_{0}$, i.e. $\Gamma_{0}=\left\{x \in \mathbb{R}^{n} \mid u_{0}(x)=0\right\}$. If $u: \mathbb{R}^{n} \times[0, \infty) \rightarrow \mathbb{R}$ is the unique (viscosity) solution of

$$
\begin{aligned}
u_{t} & =\left(\delta_{i j}-\frac{u_{x_{i}} u_{x_{j}}}{|D u|^{2}}\right) u_{x_{i} x_{j}} \quad \text { in } \mathbb{R}^{n} \times(0, \infty) \\
u(., 0) & =u_{0} \quad \text { in } \mathbb{R}^{n},
\end{aligned}
$$

we then call $\Gamma(t)=\left\{x \in \mathbb{R}^{n} \mid u(x, t)=0\right\}, t \geqslant 0$ a generalized solution of the mean curvature flow problem. Equation (1.1) is a quasilinear, degenerate and possibly singular (if $D u=0$ ) parabolic equation which gives rise to a number of difficulties both from a theoretical and numerical point of view.

Existence and uniqueness for (1.1), (1.2) have been obtained by Chen, Giga \& Goto [1] and by Evans \& Spruck [10] within the theory of viscosity solutions. The level set method therefore gives a natural way of defining a global solution for the mean curvature flow problem which is meaningful even after the onset of singularities.

We briefly describe the existence part in [10] since it is connected to the numerical method which we are going to analyse. Their idea consists of introducing the following regularized version of (1.1), (1.2), namely

$$
\begin{aligned}
u_{t}^{\epsilon} & =\left(\delta_{i j}-\frac{u_{x_{i}}^{\epsilon} u_{x_{j}}^{\epsilon}}{\epsilon^{2}+\left|D u^{\epsilon}\right|^{2}}\right) u_{x_{i} x_{j}}^{\epsilon} \quad \text { in } \mathbb{R}^{n} \times(0, \infty) \\
u^{\epsilon}(., 0) & =u_{0} \quad \text { in } \mathbb{R}^{n} .
\end{aligned}
$$


If $u_{0}$ is smooth, then (1.3), (1.4) have a global solution $u^{\epsilon}$ which satisfies

$$
\left|u^{\epsilon}\right|,\left|D u^{\epsilon}\right|,\left|u_{t}^{\epsilon}\right| \leqslant C, \quad \text { uniformly on } \mathbb{R}^{n} \times[0, \infty)
$$

where $C$ only depends on $u_{0}$. By (1.5) and the Arzela-Ascoli theorem, $\left(u^{\epsilon}\right)$ has a subsequence which converges locally uniformly on $\mathbb{R}^{n} \times[0, \infty)$. Its limit $u \in C^{0}\left(\mathbb{R}^{n} \times[0, \infty)\right)$ then is the unique viscosity solution of (1.1), (1.2) (see Section 2) and the whole sequence $\left(u^{\epsilon}\right)$ converges to $u$ as $\epsilon \rightarrow 0$.

Let us now turn to the numerical approximation of (1.1), (1.2). In [4], Crandall \& Lions gave an explicit finite difference scheme which is both monotone and consistent. Rather than discretizing (1.1), (1.2), they introduced a scheme for the approximation of (1.3), (1.4) and exploited the fact that $u^{\epsilon} \rightarrow u$ as $\epsilon \rightarrow 0$. In order to describe their method we denote by

$$
\mathcal{G}_{\rho}=\left\{\rho\left(m_{1}, \ldots, m_{n}\right) \mid m_{i} \in \mathbb{Z}, i=1, \ldots, n\right\} \quad(\rho>0)
$$

a space mesh. To every grid function $v: \mathcal{G}_{\rho} \rightarrow \mathbb{R}$ we associate a function $E v \in C^{0}\left(\mathbb{R}^{n}\right)$ satisfying

$$
(E v)(x)=v(x) \quad \text { for all } x \in \mathcal{G}_{\rho} .
$$

A simple way to construct $E v$ is the following: let

$$
Q_{n}:=\left\{q: \mathbb{R}^{n} \rightarrow \mathbb{R} \mid q(x)=\sum_{\alpha_{i} \in\{0,1\}} a_{\alpha_{1}, \ldots, \alpha_{n}} x_{1}^{\alpha_{1}} \cdots x_{n}^{\alpha_{n}}\right\} .
$$

A short calculation shows that $\operatorname{dim} Q_{n}=2^{n}$. On the other hand, the $n$-rectangle

$$
R_{m}=\left\{x \in \mathbb{R}^{n} \mid \exists 0 \leqslant \lambda_{i} \leqslant 1 \quad x=\rho m+\rho \sum_{i=1}^{n} \lambda_{i} e_{i}\right\}, \quad m \in \mathbb{Z}^{n}
$$

has $2^{n}$ vertices and it is not difficult to see that for every $v: \mathcal{G}_{\rho} \rightarrow \mathbb{R}, m \in \mathbb{Z}^{n}$ there exists a unique $\tilde{v}_{m} \in Q_{n}$ which satisfies

$$
\tilde{v}_{m}(x)=v(x) \quad \text { for all } x \in R_{m} \cap \mathcal{G}_{\rho} .
$$

Then the function $E v: \mathbb{R}^{n} \rightarrow \mathbb{R}, E v(x):=\tilde{v}_{m}(x), x \in R_{m}$ is well-defined, continuous on $\mathbb{R}^{n}$ and interpolates $v$ on $\mathcal{G}_{\rho}$. Furthermore, observing that the space of polynomials of degree less than or equal to one is contained in $Q_{n}$, the Bramble-Hilbert Lemma implies

$$
\|v-E v\|_{L^{\infty}\left(R_{m}\right)} \leqslant c \rho^{2}\left\|D^{2} v\right\|_{L^{\infty}\left(R_{m}\right)}, \quad \text { provided } v \in H^{2, \infty}\left(R_{m}\right) .
$$

The extension $E v$ allows us to evaluate a grid function on points not belonging to $\mathcal{G}_{\rho}$.

Next, we denote by $\mathcal{S}(n)$ the space of real symmetric $n \times n$ matrices. We define $\theta^{\epsilon}: \mathbb{R}^{n} \rightarrow$ $\mathcal{S}(n), \theta^{\epsilon}(p)=\left(\theta_{i k}^{\epsilon}(p)\right)_{i k}$ by

$$
\theta_{i k}^{\epsilon}(p)=\delta_{i k}-\frac{1}{1+\frac{\epsilon}{\sqrt{\epsilon^{2}+|p|^{2}}}} \frac{p_{i} p_{k}}{\epsilon^{2}+|p|^{2}} .
$$


The following identity can be viewed as an approximation of the relation $P^{2}=P$ which holds for $P=\left(\delta_{i k}-\frac{p_{i}}{|p|} \frac{p_{k}}{|p|}\right)_{i k}$ :

$$
\sum_{j=1}^{n} \theta_{i j}^{\epsilon}(p) \theta_{k j}^{\epsilon}(p)=\delta_{i k}-\frac{p_{i} p_{k}}{\epsilon^{2}+|p|^{2}}, \quad i, k=1, \ldots, n, p \in \mathbb{R}^{n} .
$$

Equation (1.8) allows us to rewrite the right-hand side of (1.3) as follows (ignoring the time dependence of $u^{\epsilon}$ for a moment):

$$
\begin{aligned}
\left(\delta_{i k}-\frac{u_{x_{i}}^{\epsilon} u_{x_{k}}^{\epsilon}}{\epsilon^{2}+\left|D u^{\epsilon}\right|^{2}}\right) u_{x_{i} x_{k}}^{\epsilon} & =\sum_{j=1}^{n}\left(\theta^{\epsilon}\left(D u^{\epsilon}\right) e_{j}\right)_{i}\left(\theta^{\epsilon}\left(D u^{\epsilon}\right) e_{j}\right) u_{k} u_{x_{i} x_{k}}^{\epsilon} \\
& \approx \sum_{j=1}^{n} \frac{u^{\epsilon}\left(.+h \theta^{\epsilon}\left(D u^{\epsilon}\right) e_{j}\right)+u^{\epsilon}\left(.-h \theta^{\epsilon}\left(D u^{\epsilon}\right) e_{j}\right)-2 u^{\epsilon}}{h^{2}}
\end{aligned}
$$

for small $h$. The above relation is the basis for the scheme in [4] which we describe now: let $\Delta t$ be a time step size and $\eta: \mathcal{G}_{\rho} \rightarrow \mathbb{R}$. Then $W_{\Delta t} \eta: \mathcal{G}_{\rho} \rightarrow \mathbb{R}$ is defined as follows:

$$
\begin{aligned}
\left(W_{\Delta t} \eta\right)(x)= & \eta(x) \\
& +\Delta t \sum_{j=1}^{n} \frac{E \eta\left(x+h \theta^{\epsilon}\left(D_{\rho} \eta(x)\right) e_{j}\right)+E \eta\left(x-h \theta^{\epsilon}\left(D_{\rho} \eta(x)\right) e_{j}\right)-2 \eta(x)}{h^{2}} \\
& +\Delta t \frac{\rho K}{h} \sum_{j=1}^{n} \frac{\eta\left(x+\rho e_{j}\right)+\eta\left(x-\rho e_{j}\right)-2 \eta(x)}{\rho^{2}} .
\end{aligned}
$$

Here, $D_{\rho} \eta(x)$ is the central difference operator, i.e.

$$
D_{\rho} \eta(x)=\frac{1}{2 \rho}\left(\eta\left(x+\rho e_{1}\right)-\eta\left(x-\rho e_{1}\right), \ldots, \eta\left(x+\rho e_{n}\right)-\eta\left(x-\rho e_{n}\right)\right) .
$$

A few remarks on the definition of $W_{\Delta t}$ are in order:

(i) Note that our definition (1.7) of $\left(\theta^{\epsilon}\right)_{i k}$ slightly differs $\left(\right.$ by the factor $\left.\left(1+\frac{\epsilon}{\sqrt{\epsilon^{2}+|p|^{2}}}\right)^{-1}\right)$ from the one given in [4]. We made this change to ensure (1.8), the results of [4], however, are not affected by this.

(ii) As mentioned above, the function $E \eta$ is introduced because $x \pm h \theta^{\epsilon}\left(D_{\rho} \eta(x)\right) e_{j}$ is not necessarily in $\mathcal{G}_{\rho}$ so that the value of a grid function at that point might not be defined.

(iii) Finally, the third term in (1.9) appears in order to guarantee monotonicity of the resulting scheme.

Let us now formulate the discrete problem: to do so, we fix $T>0$ and let $T=N \Delta t, t_{l}=l \Delta t, l=$ $0, \ldots, N$. The functions $U^{l}: \mathcal{G}_{\rho} \rightarrow \mathbb{R}$ are obtained by

$$
\begin{aligned}
U^{0}(x) & :=u_{0}(x), \quad x \in \mathcal{G}_{\rho} \\
U^{l+1}(x) & :=\left(W_{\Delta t} U^{l}\right)(x), \quad x \in \mathcal{G}_{\rho}, 0 \leqslant l \leqslant N-1 .
\end{aligned}
$$


The following convergence result for (1.10) is proved in [4].

THEOREM 1.1 Let $u$ be the viscosity solution of (1.1),(1.2) and suppose that

$$
\frac{\rho}{h^{2}} \rightarrow 0, \quad 2 n \Delta t\left(\frac{1}{h^{2}}+\frac{K}{h \rho}\right) \leqslant 1, \quad K \geqslant \frac{\sqrt{n} \operatorname{Lip}\left(u_{0}\right)}{2 \sqrt{\epsilon}}, \quad \frac{\rho K}{h} \rightarrow 0 .
$$

Then

$$
U^{l}(x)-u(x, t) \rightarrow 0 \quad \text { locally uniformly in }(x, t) \in \mathbb{R}^{n} \times[0, \infty)
$$

as $\Delta t \searrow 0$ and $l \Delta t \rightarrow t$ bounded.

When passing to the limit one thinks of $\epsilon, \rho, h$ and $K$ as being expressed in terms of $\Delta t$ subject to (1.11). The above scheme was the first one for which convergence was established. However, the question of convergence rates remained an open problem. It is this question that we want to study in this paper. Our result, Theorem 1.3 below, seems to be the first one in this direction.

There are two main difficulties which have to be overcome in order to solve this problem. First, as the above scheme approximates (1.3), (1.4) rather than (1.1), (1.2), the error between $u$ and $u^{\epsilon}$ has to be estimated in terms of $\epsilon$. We have the following result which relies on an idea communicated to us by G. Barles.

TheOREM 1.2 For every $\alpha \in\left(0, \frac{1}{2}\right), 0<T<\infty$ there is a constant $C=C\left(u_{0}, T, \alpha\right)$ such that

$$
\sup _{x \in \mathbb{R}^{n}, 0 \leqslant t \leqslant T}\left|\left(u-u^{\epsilon}\right)(x, t)\right| \leqslant C \epsilon^{\alpha}, \quad \text { for all } \epsilon>0 .
$$

Theorem 1.2 now allows us to analyse (1.9) for a fixed $\epsilon>0$. The main difficulty here consists of estimating the higher order derivatives for the solution $u^{\epsilon}$ in order to control the truncation error. While (1.5) provides uniform bounds on the solution and its first derivatives, bounds on higher order derivatives will depend upon $\epsilon$. To obtain these, we shall interpret solutions of (1.3) as entire graphs moving by mean curvature in $\mathbb{R}^{n+1}$ and exploit curvature bounds obtained by Ecker \& Huisken in [8]. Rather than listing these estimates now we shall state them at the various places when they are used. Let us now formulate our main result:

THEOREM 1.3 Suppose the following relations between the parameters appearing in the definition of $W_{\Delta t}$ hold:

$$
h=\epsilon^{\frac{5}{2}}, \quad \rho=\epsilon^{\frac{11}{2}}, \quad \Delta t=c_{1} \epsilon^{\frac{17}{2}}, \quad K=c_{2} \epsilon^{-\frac{1}{2}}
$$

for certain positive constants $c_{1}$ and $c_{2}$. Then, for every $\alpha \in\left(0, \frac{1}{2}\right), 0<T<\infty$ there is a $C=$ $C\left(u_{0}, T, \alpha\right)$ such that

$$
\sup _{x \in \mathcal{G}_{\rho}, l=0, \ldots, N}\left|U^{l}(x)-u\left(x, t_{l}\right)\right| \leqslant C \epsilon^{\alpha} .
$$

Let us review other work on the discretization of the level set equation: before existence and uniqueness for (1.1), (1.2) were yet obtained, Osher \& Sethian [18] introduced numerical schemes for motion by mean curvature and carried out calculations (cf. also [19]). In [2], Chen, Giga, Hitaka \& Honma consider a slightly different finite difference scheme and establish its $L^{\infty}$-stability. Walkington [20] proposes a finite element algorithm, proves its stability with respect to discrete $L^{\infty}$. and $W^{1,1}$-norms and presents numerical examples. Finite element calculations based on a variational formulation of (1.3) can also be found in [12]. 
Besides the level set method there are several other approaches to study motion by mean curvature: from the geometric point of view it is quite natural to use a parametrization in order to describe the evolving surfaces. This works very well before singularities occur and has the advantage that the number of space variables coincides with the dimension of the surfaces. There are several convergence results for the approximation of the curve shortening flow, see, for example, [7], [6], [14] and the references therein. For $n$ - dimensional surfaces which can be described as a graph, optimal error estimates for a semi-discrete finite element method have been obtained in [5].

Apart from the level set approach there is a further method which is capable of dealing with singularities, namely the approximation by the Allen-Cahn equation, a singularly perturbed parabolic equation. An error analysis for a fully discrete approximation to this problem has been carried out in [17].

An overview of the above methods together with an extensive list of references can be found in the survey article [9].

The paper is organized as follows: as already mentioned above, Section 2 contains the proof of Theorem 1.2. The proof of Theorem 1.3 will be given in Section 3, while the estimates for the higher order derivatives of $u^{\epsilon}$ are collected in Section 4 .

\section{Proof of Theorem 1.2}

Before we give the proof of Theorem 1.2 we would like to recall the notion of a viscosity solution for (1.1). Let us begin by introducing the parabolic second order superjets $\mathcal{P}^{2,+} u(x, t)$ and $\mathcal{P}^{2,-} u(x, t)$. We set

$$
\begin{aligned}
\mathcal{P}^{2,+} u(x, t):= & \left\{(a, \xi, X) \in \mathbb{R} \times \mathbb{R}^{n} \times \mathcal{S}(n) \mid u(y, s) \leqslant u(x, t)+a(s-t)+\langle\xi, y-x\rangle\right. \\
& \left.+\frac{1}{2}\langle X(y-x), y-x\rangle+o\left(|s-t|+|y-x|^{2}\right) \text { as }(y, s) \rightarrow(x, t)\right\}
\end{aligned}
$$

and $\mathcal{P}^{2,-} u(x, t)=-\mathcal{P}^{2,+}(-u)(x, t)$. The closure of $\mathcal{P}^{2,+} u(x, t)$ is defined by

$$
\begin{aligned}
\overline{\mathcal{P}}^{2,+} u(x, t):= & \left\{(a, \xi, X) \in \mathbb{R} \times \mathbb{R}^{n} \times \mathcal{S}(n) \mid \exists\left(x_{m}, t_{m}, a_{m}, \xi_{m}, X_{m}\right)\right. \\
& \in \mathbb{R}^{n} \times[0, \infty) \times \mathbb{R} \times \mathbb{R}^{n} \times \mathcal{S}(n) \text { with }\left(a_{m}, \xi_{m}, X_{m}\right) \in \mathcal{P}^{2,+} u\left(x_{m}, t_{m}\right) \text { and } \\
& \left.\left(x_{m}, t_{m}, a_{m}, \xi_{m}, X_{m}\right) \rightarrow(x, t, a, \xi, X), m \rightarrow \infty\right\}
\end{aligned}
$$

and similarly for $\overline{\mathcal{P}}^{2,-} u(x, t)$.

Definition 2.1 A function $u \in C^{0}\left(\mathbb{R}^{n} \times[0, \infty)\right)$ is called a viscosity subsolution of (1.1) provided that for every $\left(x_{0}, t_{0}\right) \in \mathbb{R}^{n} \times(0, \infty)$ and every $(a, \xi, X) \in \overline{\mathcal{P}}^{2,+} u(x, t)$

$$
\begin{array}{ll}
a \leqslant\left(\delta_{i j}-\frac{\xi_{i} \xi_{j}}{|\xi|^{2}}\right) X_{i j} & \text { if } \xi \neq 0 \\
a \leqslant\left(\delta_{i j}-p_{i} p_{j}\right) X_{i j} & \text { for some }|p| \leqslant 1, \text { if } \xi=0 .
\end{array}
$$

A viscosity supersolution is defined analogously; $\overline{\mathcal{P}}^{2,+} u(x, t)$ is replaced by $\overline{\mathcal{P}}^{2,-} u(x, t)$ and $\leqslant$ by $\geqslant$. A viscosity solution of (1.1) is a function $u \in C^{0}\left(\mathbb{R}^{n} \times[0, \infty)\right)$ which is both a subsolution and a supersolution.

In what follows we shall assume that the initial function $u_{0}$ satisfies

$$
u_{0} \in C^{4}\left(\mathbb{R}^{n}\right) \quad \text { and } \quad u_{0}(x)=1, \quad|x| \geqslant S
$$


for some $S>0$. The results of [10] imply that (1.1), (1.2) have a unique viscosity solution $u \in$ $C^{0}\left(\mathbb{R}^{n} \times[0, \infty)\right)$ such that

$$
\|u\|_{W^{1, \infty}\left(\mathbb{R}^{n} \times(0, \infty)\right)} \leqslant C \quad \text { and } \quad u(x, t)=1, \quad|x|+t \geqslant R
$$

for some $R>0$ depending only on $S$. Furthermore, the solutions $\left(u^{\epsilon}\right)$ of (1.3), (1.4) converge locally uniformly to $u$. Our aim in this section is to establish a rate for this convergence as stated in Theorem 1.2.

To begin, let us fix $\alpha \in\left(0, \frac{1}{2}\right)$ and $0<T<\infty$. We claim that there exists a constant $M=$ $M\left(u_{0}, T, \alpha\right)$ such that

$$
\sup _{x \in \mathbb{R}^{n}, 0 \leqslant t \leqslant T}\left(u-u^{\epsilon}\right)(x, t) \leqslant M \epsilon^{\alpha} \quad \text { for all } \epsilon>0 .
$$

We shall argue by contradiction. Let us assume that for every $M \geqslant 0$ there exists $\epsilon=\epsilon(M)>0$ such that

$$
\sup _{x \in \mathbb{R}^{n}, 0 \leqslant t \leqslant T}\left(u-u^{\epsilon}\right)(x, t)>M \epsilon^{\alpha} .
$$

We define $\gamma \in \mathbb{R}$ by

$$
\gamma=\frac{2(1-\alpha)}{1-2 \alpha}>2
$$

and consider the function $w: \mathbb{R}^{n} \times \mathbb{R}^{n} \times[0, T] \rightarrow \mathbb{R}$,

$$
w(x, y, t):=u(x, t)-u^{\epsilon}(y, t)-\frac{\mu}{\gamma} \epsilon^{1-\frac{\gamma}{2}}|x-y|^{\gamma}-\frac{M}{2 T} \epsilon^{\alpha} t .
$$

Note that in view of (2.4)

$$
\begin{aligned}
\sup _{x \in \mathbb{R}^{n}, y \in \mathbb{R}^{n}, 0 \leqslant t \leqslant T} w(x, y, t) \geqslant & \sup _{x \in \mathbb{R}^{n}, 0 \leqslant t \leqslant T}\left(u-u^{\epsilon}\right)(x, t)-\frac{M}{2 T} \epsilon^{\alpha} T \\
& >\frac{M}{2} \epsilon^{\alpha} .
\end{aligned}
$$

Next, let us make sure that sup $w$ is actually attained. Since

$$
\begin{aligned}
w(x, y, t) & \leqslant \sup _{\mathbb{R}^{n} \times[0, T]} u+\sup _{\mathbb{R}^{n} \times[0, T]}\left(-u^{\epsilon}\right)-\frac{\mu}{\gamma} \epsilon^{1-\frac{\gamma}{2}}|x-y|^{\gamma} \\
& \leqslant C-\frac{\mu}{\gamma} \epsilon^{1-\frac{\gamma}{2}}|x-y|^{\gamma} \\
& \leqslant \frac{M}{4} \epsilon^{\alpha}
\end{aligned}
$$

provided

$$
|x-y| \geqslant\left(\frac{\gamma C}{\mu} \epsilon^{\frac{\gamma}{2}-1}-\frac{M \gamma}{4 \mu} \epsilon^{\alpha+\left(\frac{\gamma}{2}-1\right)}\right)^{\frac{1}{\gamma}}=: r
$$

we deduce

$$
\sup _{x \in \mathbb{R}^{n}, y \in \mathbb{R}^{n}, 0 \leqslant t \leqslant T} w(x, y, t)=\sup _{|x-y| \leqslant r, 0 \leqslant t \leqslant T} w(x, y, t) .
$$


Furthermore, from step 1 in the proof of Lemma 2.3 in [11] we conclude that there exist $B_{\epsilon}>$ $0, b_{\epsilon}>0$ such that

$$
\left|1-u^{\epsilon}(x, t)\right| \leqslant B_{\epsilon} e^{-b_{\epsilon}|x|^{2}} \leqslant \frac{B_{\epsilon}}{b_{\epsilon}} \frac{1}{|x|^{2}}, \quad x \neq 0 .
$$

Combining this estimate with (2.2) we obtain for $|x| \geqslant \tilde{R}:=\max \left(R, r+\sqrt{\frac{4 B_{\epsilon}}{M b_{\epsilon}} \epsilon^{-\alpha}}\right)$ and $|x-y| \leqslant r$

$$
w(x, y, t) \leqslant\left|1-u^{\epsilon}(y, t)\right| \leqslant \frac{B_{\epsilon}}{b_{\epsilon}} \frac{1}{|y|^{2}} \leqslant \frac{B_{\epsilon}}{b_{\epsilon}} \frac{1}{(\tilde{R}-r)^{2}} \leqslant \frac{M}{4} \epsilon^{\alpha}
$$

so that

$$
\sup _{x \in \mathbb{R}^{n}, y \in \mathbb{R}^{n}, 0 \leqslant t \leqslant T} w(x, y, t)=\sup _{|x| \leqslant \tilde{R},|x-y| \leqslant r, 0 \leqslant t \leqslant T} w(x, y, t)=w(\hat{x}, \hat{y}, \hat{t})
$$

for some $(\hat{x}, \hat{y}, \hat{t})$. We claim that $\hat{t}>0$ provided $\mu$ is suitably chosen. To see this, compute

$$
\begin{aligned}
w(x, y, 0) & =u_{0}(x)-u_{0}(y)-\frac{\mu}{\gamma} \epsilon^{1-\frac{\gamma}{2}}|x-y|^{\gamma} \\
& \leqslant|x-y|\left(\operatorname{Lip}\left(u_{0}\right)-\frac{\mu}{\gamma} \epsilon^{1-\frac{\gamma}{2}}|x-y|^{\gamma-1}\right)
\end{aligned}
$$

and distinguish two cases: if $|x-y|<\frac{1}{4 \operatorname{Lip}\left(u_{0}\right)} M \epsilon^{\alpha}$, then (2.7) implies

$$
w(x, y, 0) \leqslant \frac{M}{4} \epsilon^{\alpha} .
$$

If, on the other hand $|x-y| \geqslant \frac{1}{4 \operatorname{Lip}\left(u_{0}\right)} M \epsilon^{\alpha}$, we again use (2.7) to conclude

$$
\begin{aligned}
w(x, y, 0) & \leqslant|x-y|\left(\operatorname{Lip}\left(u_{0}\right)-\frac{\mu}{\gamma} \epsilon^{1-\frac{\gamma}{2}}\left(\frac{M \epsilon^{\alpha}}{4 \operatorname{Lip}\left(u_{0}\right)}\right)^{\gamma-1}\right) \\
& =|x-y|\left(\operatorname{Lip}\left(u_{0}\right)-\frac{\mu}{\gamma}\left(\frac{M}{4 \operatorname{Lip}\left(u_{0}\right)}\right)^{\gamma-1}\right)
\end{aligned}
$$

since $1-\frac{\gamma}{2}+\alpha(\gamma-1)=0$ by (2.5). If we chose $\mu$ in such a way that $4^{\gamma-1} \gamma \operatorname{Lip}\left(u_{0}\right)^{\gamma} \leqslant \mu M^{\gamma-1}$, say,

$$
\mu=\frac{\gamma 4^{\gamma} \operatorname{Lip}\left(u_{0}\right)^{\gamma}}{M^{\gamma-1}}
$$

we obtain $w(x, y, 0) \leqslant 0$ so that in conclusion

$$
\sup _{x \in \mathbb{R}^{n}, y \in \mathbb{R}^{n}} w(x, y, 0)<\frac{M}{4} \epsilon^{\alpha}
$$

which shows that $\hat{t}>0$. In addition, we may assume that $\hat{t}<T$. Otherwise, we could replace $u$ by $\tilde{u}(x, t):=u(x, t)-\frac{\delta}{T-t}(\delta>0)$, which satisfies $\lim _{t \nearrow T} \tilde{u}(x, t)=-\infty$ uniformly in $x \in \mathbb{R}^{n}$. 
Furthermore, $\tilde{u}$ is a subsolution of (1.1) which is sufficient for the subsequent analysis (see (2.11)). This would allow us to prove (2.3) for $\tilde{u}$ in place of $u$ and the result then follows by sending $\delta \rightarrow 0$. Summing up, there exists $(\hat{x}, \hat{y}, \hat{t}) \in \mathbb{R}^{n} \times \mathbb{R}^{n} \times(0, T)$ such that

$$
\sup _{x \in \mathbb{R}^{n}, y \in \mathbb{R}^{n}, 0 \leqslant t \leqslant T} w(x, y, t)=w(\hat{x}, \hat{y}, \hat{t}) .
$$

In order to proceed, we write $w$ in the form

$$
w(x, y, t)=u(x, t)-u^{\epsilon}(y, t)-\phi(x, y, t)
$$

where

$$
\phi(x, y, t)=\frac{\mu}{\gamma} \epsilon^{1-\frac{\gamma}{2}}|x-y|^{\gamma}+\frac{M}{2 T} \epsilon^{\alpha} t .
$$

By Theorem 8.3 in [3] there exist for every $\rho>0$ matrices $X, Y \in \mathcal{S}(n)$ such that

$$
\begin{aligned}
& \left(a, D_{x} \phi(\hat{x}, \hat{y}, \hat{t}), X\right) \in \overline{\mathcal{P}}^{2,+} u(\hat{x}, \hat{t}) \\
& \left(b, D_{y} \phi(\hat{x}, \hat{y}, \hat{t}), Y\right) \in \overline{\mathcal{P}}^{2,+}\left(-u^{\epsilon}(\hat{y}, \hat{t})\right)=-\overline{\mathcal{P}}^{2,-} u^{\epsilon}(\hat{y}, \hat{t})
\end{aligned}
$$

$$
a+b=\frac{M}{2 T} \epsilon^{\alpha}
$$

$$
-\left(\frac{1}{\rho}+\|A\|\right) I \leqslant\left(\begin{array}{cc}
X & 0 \\
0 & Y
\end{array}\right) \leqslant A+\rho A^{2}
$$

where $A=D_{(x, y)}^{2} \phi(\hat{x}, \hat{y}, \hat{t})$. Letting $\hat{\xi}=\hat{x}-\hat{y}$, a short computation gives

$$
\begin{gathered}
D_{x} \phi(\hat{x}, \hat{y}, \hat{t})=-D_{y} \phi(\hat{x}, \hat{y}, \hat{t})=\mu \epsilon^{1-\frac{\gamma}{2}}|\hat{\xi}|^{\gamma-2} \hat{\xi} \\
A=\left(\begin{array}{cc}
B & -B \\
-B & B
\end{array}\right), \quad B=\mu \epsilon^{1-\frac{\gamma}{2}}|\hat{\xi}|^{\gamma-4}\left((\gamma-2) \hat{\xi} \otimes \hat{\xi}+|\hat{\xi}|^{2} I\right) .
\end{gathered}
$$

Here, $\otimes$ denotes the tensor product of two vectors in $\mathbb{R}^{n}$. In view of (iii) we have for all $\eta \in \mathbb{R}^{n}$

$$
\begin{aligned}
\eta^{t}(X+Y) \eta & =\left(\eta^{t}, \eta^{t}\right)\left(\begin{array}{cc}
X & 0 \\
0 & Y
\end{array}\right)\left(\begin{array}{l}
\eta \\
\eta
\end{array}\right) \\
& \leqslant\left(\eta^{t}, \eta^{t}\right)\left(\left(\begin{array}{cc}
B & -B \\
-B & B
\end{array}\right)+\rho\left(\begin{array}{cc}
2 B^{2} & -2 B^{2} \\
-2 B^{2} & 2 B^{2}
\end{array}\right)\right)\left(\begin{array}{l}
\eta \\
\eta
\end{array}\right)=0,
\end{aligned}
$$

so that $X+Y \leqslant 0$

Next, since $\left(a, D_{x} \phi(\hat{x}, \hat{y}, \hat{t}), X\right) \in \overline{\mathcal{P}}^{2,+} u(\hat{x}, \hat{t})$ and $u$ is a viscosity subsolution of (1.1), we obtain

$$
\begin{aligned}
& a-\left(\delta_{i j}-\frac{\hat{\xi}_{i} \hat{\xi}_{j}}{|\hat{\xi}|^{2}}\right) X_{i j} \leqslant 0, \quad \text { if } \hat{\xi} \neq 0 \\
& a-\left(\delta_{i j}-p_{i} p_{j}\right) X_{i j} \leqslant 0, \quad \text { for some }|p| \leqslant 1, \text { if } \hat{\xi}=0 .
\end{aligned}
$$


Furthermore,

$$
u_{t}^{\epsilon}(\hat{y}, \hat{t})=-b, \quad D u^{\epsilon}(\hat{y}, \hat{t})=-D_{y} \phi(\hat{x}, \hat{y}, \hat{t}), \quad D^{2} u^{\epsilon}(\hat{y}, \hat{t}) \geqslant-Y
$$

because $u^{\epsilon}$ is smooth. Thus (1.3) and (2.9) imply

$$
\begin{aligned}
b=-u_{t}^{\epsilon}(\hat{y}, \hat{t}) & =-\left(\delta_{i j}-\frac{u_{y_{i}}^{\epsilon}(\hat{y}, \hat{t}) u_{y_{j}}^{\epsilon}(\hat{y}, \hat{t})}{\epsilon^{2}+\left|D u^{\epsilon}(\hat{y}, \hat{t})\right|^{2}}\right) u_{y_{i} y_{j}}^{\epsilon}(\hat{y}, \hat{t}) \\
& \leqslant\left(\delta_{i j}-\frac{\mu^{2} \epsilon^{2-\gamma}|\hat{\xi}|^{2 \gamma-4} \hat{\xi}_{i} \hat{\xi}_{j}}{\epsilon^{2}+\mu^{2} \epsilon^{2-\gamma}|\hat{\xi}|^{2 \gamma-2}}\right) Y_{i j} .
\end{aligned}
$$

We distinguish two cases: if $\hat{\xi} \neq 0$, (ii), (2.11) and (2.12) give

$$
\begin{aligned}
\frac{M}{2 T} \epsilon^{\alpha} & =a+b \\
& \leqslant\left(\delta_{i j}-\frac{\hat{\xi}_{i} \hat{\xi}_{j}}{|\hat{\xi}|^{2}}\right) X_{i j}+\left(\delta_{i j}-\frac{\mu^{2} \epsilon^{2-\gamma}|\hat{\xi}|^{2 \gamma-4} \hat{\xi}_{i} \hat{\xi}_{j}}{\left.\epsilon^{2}+\mu^{2} \epsilon^{2-\gamma|\hat{\xi}|^{2 \gamma-2}}\right) Y_{i j}}\right. \\
& =\left(\delta_{i j}-\frac{\hat{\xi}_{i} \hat{\xi}_{j}}{|\hat{\xi}|^{2}}\right)\left(X_{i j}+Y_{i j}\right)+\frac{\epsilon^{2} \hat{\xi}^{t} Y \hat{\xi}}{|\hat{\xi}|^{2}\left(\epsilon^{2}+\mu^{2} \epsilon^{2-\gamma}|\hat{\xi}|^{2 \gamma-2}\right)} \\
& \leqslant \frac{\epsilon^{2} \hat{\xi}^{t} Y \hat{\xi}}{|\hat{\xi}|^{2}\left(\epsilon^{2}+\mu^{2} \epsilon^{\left.2-\gamma|\hat{\xi}|^{2 \gamma-2}\right)} .\right.}
\end{aligned}
$$

since $X+Y \leqslant 0$. In order to estimate this expression, we observe that in view of (iii) and (2.10)

$$
\begin{aligned}
\hat{\xi}^{t} Y \hat{\xi} & =\left(0, \hat{\xi}^{t}\right)\left(\begin{array}{cc}
X & 0 \\
0 & Y
\end{array}\right)\left(\begin{array}{c}
0 \\
\hat{\xi}
\end{array}\right) \leqslant \hat{\xi}^{t} B \hat{\xi}+2 \rho \hat{\xi}^{t} B^{2} \hat{\xi} \\
& =\mu(\gamma-1) \epsilon^{1-\frac{\gamma}{2}}|\hat{\xi}|^{\gamma}+2 \rho \hat{\xi}^{t} B^{2} \hat{\xi} .
\end{aligned}
$$

Inserting this inequality into (2.13) and letting $\rho \rightarrow 0$ we obtain

$$
\begin{aligned}
\frac{M}{2 T} \epsilon^{\alpha} & \leqslant \mu(\gamma-1) \frac{\epsilon^{3-\frac{\gamma}{2}}|\hat{\xi}|^{\gamma-2}}{\epsilon^{2}+\mu^{2} \epsilon^{2-\gamma}|\hat{\xi}|^{2 \gamma-2}} \\
& =(\gamma-1) \mu^{\frac{1}{\gamma-1}} \epsilon^{\frac{\gamma-2}{2(\gamma-1)}} \frac{\epsilon^{\frac{\gamma}{\gamma-1}} \mu^{\frac{\gamma-2}{\gamma-1}} \epsilon^{-\frac{(\gamma-2)^{2}}{2(\gamma-1)}}|\hat{\xi}|^{\gamma-2}}{\epsilon^{2}+\mu^{2} \epsilon^{2-\gamma}|\hat{\xi}|^{2 \gamma-2}} .
\end{aligned}
$$

Applying Young's inequality $a b \leqslant \frac{1}{p} a^{p}+\frac{1}{q} b^{q}\left(\frac{1}{p}+\frac{1}{q}=1\right)$ with $p=\frac{2(\gamma-1)}{\gamma}$ and $q=$ $\frac{2(\gamma-1)}{\gamma-2}$ we deduce

$$
\frac{M}{2 T} \epsilon^{\alpha} \leqslant(\gamma-1) \mu^{\frac{1}{\gamma-1}} \epsilon^{\frac{\gamma-2}{2(\gamma-1)}} \frac{\frac{\gamma}{2(\gamma-1)} \epsilon^{2}+\frac{\gamma-2}{2(\gamma-1)} \mu^{2} \epsilon^{2-\gamma}|\hat{\xi}|^{2 \gamma-2}}{\epsilon^{2}+\mu^{2} \epsilon^{2-\gamma}|\hat{\xi}|^{2 \gamma-2}}
$$




$$
\begin{aligned}
& \leqslant C(\gamma) \mu^{\frac{1}{\gamma-1}} \epsilon^{\frac{\gamma-2}{2(\gamma-1)}} \\
& =C(\gamma)\left(\frac{\gamma 4^{\gamma} \operatorname{Lip}\left(u_{0}\right)^{\gamma}}{M^{\gamma-1}}\right)^{\frac{1}{\gamma-1}} \epsilon^{\alpha} \\
& =C\left(\gamma, u_{0}\right) \epsilon^{\alpha} M^{-1}
\end{aligned}
$$

where we used (2.8) and (2.5). This leads to a contradiction if $M$ is large.

Finally, it remains to consider the case $\hat{\xi}=0$. Now, (2.11) and (2.12) give

$$
\begin{aligned}
\frac{M}{2 T} \epsilon^{\alpha} & =a+b \\
& \leqslant\left(\delta_{i j}-p_{i} p_{j}\right) X_{i j}+\delta_{i j} Y_{i j} \\
& =\left(\delta_{i j}-p_{i} p_{j}\right)\left(X_{i j}+Y_{i j}\right)+p^{t} Y p .
\end{aligned}
$$

Since $\gamma>2,(2.10)$ implies that $B=0$ so that the calculation in (2.14) yields $p^{t} Y p \leqslant 0$. Furthermore, since $X+Y \leqslant 0$ we obtain $\frac{M}{2 T} \epsilon^{\alpha} \leqslant 0$ which is again a contradiction. Thus (2.3) is proved. The estimate for $\sup \left(u^{\epsilon}-u\right)$ follows in an analogous way, which concludes the proof of Theorem 1.2.

\section{Proof of the error estimate}

In this section we shall prove our main result, Theorem 1.3. The analysis of the finite difference scheme requires, as usual, arguments based on Taylor expansions which in our case means that we have to estimate higher order derivatives of $u^{\epsilon}$. The proofs for these estimates are postponed to Section 4.

To begin, let $W_{\Delta t}$ be given by (1.9), i.e. $W_{\Delta t}=W_{1, \Delta t}+W_{2, \Delta t}$, where for $x \in \mathcal{G}_{\rho}$

$$
\begin{aligned}
\left(W_{1, \Delta t} \eta\right)(x)= & \eta(x) \\
& +\Delta t \sum_{j=1}^{n} \frac{(E \eta)\left(x+h \theta^{\epsilon}\left(D_{\rho} \eta(x)\right) e_{j}\right)+(E \eta)\left(x-h \theta^{\epsilon}\left(D_{\rho} \eta(x)\right) e_{j}\right)-2 \eta(x)}{h^{2}} \\
\left(W_{2, \Delta t} \eta\right)(x)= & \Delta t \frac{\rho K}{h} \sum_{j=1}^{n} \frac{\eta\left(x+\rho e_{j}\right)+\eta\left(x-\rho e_{j}\right)-2 \eta(x)}{\rho^{2}}
\end{aligned}
$$

and $E \eta$ was introduced in Section 1 . We start by estimating the local truncation error for $W_{1, \Delta t}$.

LEMma 3.1 Let $u^{\epsilon}$ be the solution of (1.3), (1.4). For every $\alpha \in\left(0, \frac{1}{2}\right)$ there exist constants $C \geqslant 0$ and $\lambda>0$ which only depend on $u_{0}, T, \alpha$ and $n$ such that

$$
\begin{aligned}
\sup _{x \in \mathcal{G}_{\rho}, l=0, \ldots, N-1}\left|u^{\epsilon}\left(x, t_{l+1}\right)-\left(W_{1, \Delta t} u^{\epsilon}\left(., t_{l}\right)\right)(x)\right| \\
\quad \leqslant C \Delta t\left(\rho^{2} h^{-2} \epsilon^{-2}+\rho^{2} \epsilon^{-6} h^{-1}+h^{2 \alpha} \epsilon^{-4 \alpha}+\Delta t \epsilon^{-3}\right)
\end{aligned}
$$

provided $h \leqslant \lambda \epsilon^{2}$. 
Proof. Let us fix $x \in \mathcal{G}_{\rho}$ and $l \in\{0, \ldots, N-1\}$. Clearly,

$$
\begin{aligned}
& \left(W_{1, \Delta t} u^{\epsilon}\left(., t_{l}\right)\right)(x)=u^{\epsilon}\left(x, t_{l}\right)+R^{\epsilon}\left(x, t_{l}\right) \\
& \quad+\Delta t \sum_{j=1}^{n} \frac{u^{\epsilon}\left(x+h \theta^{\epsilon}\left(D u^{\epsilon}\left(x, t_{l}\right)\right) e_{j}, t_{l}\right)+u^{\epsilon}\left(x-h \theta^{\epsilon}\left(D u^{\epsilon}\left(x, t_{l}\right)\right) e_{j}, t_{l}\right)-2 u^{\epsilon}\left(x, t_{l}\right)}{h^{2}}
\end{aligned}
$$

where

$$
\begin{aligned}
R^{\epsilon}\left(x, t_{l}\right)= & \frac{\Delta t}{h^{2}} \sum_{j=1}^{n}\left(\left(E u^{\epsilon}\left(., t_{l}\right)\right)\left(x+h \theta^{\epsilon}\left(D_{\rho} u^{\epsilon}\left(x, t_{l}\right)\right) e_{j}\right)-u^{\epsilon}\left(x+h \theta^{\epsilon}\left(D u^{\epsilon}\left(x, t_{l}\right)\right) e_{j}, t_{l}\right)\right. \\
& \left.+\left(E u^{\epsilon}\left(., t_{l}\right)\right)\left(x-h \theta^{\epsilon}\left(D_{\rho} u^{\epsilon}\left(x, t_{l}\right)\right) e_{j}\right)-u^{\epsilon}\left(x-h \theta^{\epsilon}\left(D u^{\epsilon}\left(x, t_{l}\right)\right) e_{j}, t_{l}\right)\right) .
\end{aligned}
$$

The remainder term is estimated by

$$
\begin{aligned}
& \left|R^{\epsilon}\left(x, t_{l}\right)\right| \\
& \quad \leqslant C \Delta t h^{-2}\left(\left\|\left(u^{\epsilon}-E u^{\epsilon}\right)\left(., t_{l}\right)\right\|_{L^{\infty}}+h\left\|D u^{\epsilon}\right\|_{\left.L^{\infty}\left|\theta^{\epsilon}\left(D_{\rho} u^{\epsilon}\left(x, t_{l}\right)\right)-\theta^{\epsilon}\left(D u^{\epsilon}\left(x, t_{l}\right)\right)\right|\right)}\right. \\
& \quad \leqslant C \Delta t h^{-2}\left(\rho^{2}\left\|D^{2} u^{\epsilon}\left(., t_{l}\right)\right\|_{L^{\infty}}+h\left\|D \theta^{\epsilon}\right\|_{\left.\left.L^{\infty} \mid D_{\rho} u^{\epsilon}\left(x, t_{l}\right)-D u^{\epsilon}\left(x, t_{l}\right)\right) \mid\right) .}\right.
\end{aligned}
$$

Here we used (1.6) and (1.5). Next, it is not difficult to see that

$$
\left|\frac{\partial \theta_{i k}^{\epsilon}}{\partial p_{j}}(p)\right| \leqslant \frac{C}{\epsilon}, \quad i, j, k=1, \ldots, n, \quad p \in \mathbb{R}^{n} .
$$

Furthermore,

$$
\left|D_{\rho} u^{\epsilon}\left(x, t_{l}\right)-D u^{\epsilon}\left(x, t_{l}\right)\right| \leqslant C \rho^{2}\left\|D^{3} u^{\epsilon}\left(., t_{l}\right)\right\|_{L^{\infty}}
$$

so that we obtain, by Corollary 4.2 and Lemma 4.4,

$$
\begin{aligned}
\left|R^{\epsilon}\left(x, t_{l}\right)\right| & \leqslant C \Delta t h^{-2}\left(\rho^{2}\left\|D^{2} u^{\epsilon}\left(., t_{l}\right)\right\|_{L^{\infty}}+h \epsilon^{-1} \rho^{2}\left\|D^{3} u^{\epsilon}\left(., t_{l}\right)\right\|_{L^{\infty}}\right) \\
& \leqslant C \Delta t h^{-2}\left(\rho^{2} \epsilon^{-2}+h \rho^{2} \epsilon^{-6}\right) .
\end{aligned}
$$

Next, let us define $P^{\epsilon}:=\theta^{\epsilon}\left(D u^{\epsilon}\left(x, t_{l}\right)\right) \in \mathcal{S}(n)$. A Taylor expansion gives for $j=1, \ldots, n$

$$
\begin{aligned}
u^{\epsilon}\left(x \pm h P^{\epsilon} e_{j}, t_{l}\right)= & u^{\epsilon}\left(x, t_{l}\right) \pm h\left\langle D u^{\epsilon}\left(x, t_{l}\right), P^{\epsilon} e_{j}\right\rangle \\
& +\frac{1}{2} h^{2}\left\langle D^{2} u^{\epsilon}\left(x \pm \sigma_{j}^{ \pm} P^{\epsilon} e_{j}, t_{l}\right) P^{\epsilon} e_{j}, P^{\epsilon} e_{j}\right\rangle
\end{aligned}
$$

where $\sigma_{j}^{ \pm} \in(0, h)$. Then

$$
\sum_{j=1}^{n} \frac{u^{\epsilon}\left(x+h P^{\epsilon} e_{j}, t_{l}\right)+u^{\epsilon}\left(x-h P^{\epsilon} e_{j}, t_{l}\right)-2 u^{\epsilon}\left(x, t_{l}\right)}{h^{2}}
$$




$$
\begin{aligned}
= & \sum_{j=1}^{n}\left\langle D^{2} u^{\epsilon}\left(x, t_{l}\right) P^{\epsilon} e_{j}, P^{\epsilon} e_{j}\right\rangle \\
& +\frac{1}{2} \sum_{j=1}^{n}\left\langle\left(D^{2} u^{\epsilon}\left(x+\sigma_{j}^{+} P^{\epsilon} e_{j}, t_{l}\right)-D^{2} u^{\epsilon}\left(x, t_{l}\right)\right) P^{\epsilon} e_{j}, P^{\epsilon} e_{j}\right\rangle \\
& +\frac{1}{2} \sum_{j=1}^{n}\left\langle\left(D^{2} u^{\epsilon}\left(x-\sigma_{j}^{-} P^{\epsilon} e_{j}, t_{l}\right)-D^{2} u^{\epsilon}\left(x, t_{l}\right)\right) P^{\epsilon} e_{j}, P^{\epsilon} e_{j}\right\rangle .
\end{aligned}
$$

Using (1.8) and (1.3) we may rewrite the first term as

$$
\begin{aligned}
\sum_{j=1}^{n}\left\langle D^{2} u^{\epsilon}\left(x, t_{l}\right) P^{\epsilon} e_{j}, P^{\epsilon} e_{j}\right\rangle & =\sum_{j=1}^{n} \theta_{i j}^{\epsilon}\left(D u^{\epsilon}\left(x, t_{l}\right)\right) \theta_{k j}^{\epsilon}\left(D u^{\epsilon}\left(x, t_{l}\right)\right) u_{x_{i} x_{k}}^{\epsilon}\left(x, t_{l}\right) \\
& =\left(\delta_{i k}-\frac{u_{x_{i}}^{\epsilon}\left(x, t_{l}\right) u_{x_{k}}^{\epsilon}\left(x, t_{l}\right)}{\epsilon^{2}+\left|D u^{\epsilon}\left(x, t_{l}\right)\right|^{2}}\right) u_{x_{i} x_{k}}^{\epsilon}\left(x, t_{l}\right) \\
& =u_{t}^{\epsilon}\left(x, t_{l}\right) .
\end{aligned}
$$

Inserting these identities into (3.1) gives

$$
\left(W_{1, \Delta t} u^{\epsilon}\left(., t_{l}\right)\right)(x)=u^{\epsilon}\left(x, t_{l}\right)+\Delta t u_{t}^{\epsilon}\left(x, t_{l}\right)+R^{\epsilon}\left(x, t_{l}\right)+\tilde{R}^{\epsilon}\left(x, t_{l}\right)
$$

where, by Lemma 4.8 , since $2 \alpha<1$

$$
\begin{aligned}
\left|\tilde{R}^{\epsilon}\left(x, t_{l}\right)\right| & \leqslant C \Delta t \sup _{1 \leqslant j \leqslant n|\sigma| \leqslant h} \sup _{1}\left|\left\langle\left(D^{2} u^{\epsilon}\left(x+\sigma P^{\epsilon} e_{j}, t_{l}\right)-D^{2} u^{\epsilon}\left(x, t_{l}\right)\right) P^{\epsilon} e_{j}, P^{\epsilon} e_{j}\right\rangle\right| \\
& \leqslant C \Delta t h^{2 \alpha} \epsilon^{-4 \alpha}
\end{aligned}
$$

uniformly in $x \in \mathcal{G}_{\rho}, l \in\{0, \ldots, N-1\}$, provided $h \leqslant \lambda \epsilon^{2}$.

Finally, observing that

$$
u^{\epsilon}\left(x, t_{l+1}\right)=u^{\epsilon}\left(x, t_{l}\right)+\Delta t u_{t}^{\epsilon}\left(x, t_{l}\right)+\int_{t_{l}}^{t_{l+1}}\left(t_{l+1}-s\right) u_{t t}^{\epsilon}(x, s) \mathrm{d} s
$$

we obtain from (3.4)

$$
\left|\left(W_{1, \Delta t} u^{\epsilon}\left(., t_{l}\right)\right)(x)-u^{\epsilon}\left(x, t_{l+1}\right)\right| \leqslant\left|R^{\epsilon}\left(x, t_{l}\right)\right|+\left|\tilde{R}^{\epsilon}\left(x, t_{l}\right)\right|+(\Delta t)^{2}\left\|u_{t t}^{\epsilon}\right\|_{L^{\infty}} .
$$

The lemma now follows from our estimates on the remainder terms and Corollary 4.6.

LEMMA 3.2 Let $u^{\epsilon}$ be the solution of (1.3), (1.4). Then

$$
\left|\left(W_{2, \Delta t} u^{\epsilon}\left(., t_{l}\right)\right)(x)\right| \leqslant C \Delta t K \rho h^{-1} \epsilon^{-2}, \quad x \in \mathcal{G}_{\rho}, l=0, \ldots, N .
$$

Proof. A Taylor expansion gives for $j=1, \ldots, n$

$$
\frac{u^{\epsilon}\left(x+\rho e_{j}, t_{l}\right)+u^{\epsilon}\left(x-\rho e_{j}, t_{l}\right)-2 u^{\epsilon}\left(x, t_{l}\right)}{\rho^{2}}=\frac{1}{2}\left(u_{x_{j} x_{j}}^{\epsilon}\left(\xi_{j}^{+}, t_{l}\right)+u_{x_{j} x_{j}}^{\epsilon}\left(\xi_{j}^{-}, t_{l}\right)\right)
$$

for some $\xi_{j}^{ \pm} \in\left(x, x \pm \rho e_{j}\right)$. The result then follows from Corollary 4.2. 
We are now in position to prove Theorem 1.3. Let us fix $\alpha \in\left(0, \frac{1}{2}\right), 0<T<\infty$ and define

$$
L:=L(\epsilon, \Delta t, \rho, h, K):=\rho^{2} h^{-2} \epsilon^{-2}+\rho^{2} h^{-1} \epsilon^{-6}+h^{2 \alpha} \epsilon^{-4 \alpha}+\Delta t \epsilon^{-3}+K \rho h^{-1} \epsilon^{-2} .
$$

Combining Lemma 3.1 and 3.2 we obtain

$$
\left|\left(W_{\Delta t} u^{\epsilon}\left(., t_{l}\right)\right)(x)-u^{\epsilon}\left(x, t_{l+1}\right)\right| \leqslant C \Delta t L, \quad \text { if } h \leqslant \lambda \epsilon^{2} .
$$

In addition, the results of Section 1.3 in [4] ensure that $W_{\Delta t}$ is monotone (i.e. $f \leqslant g$ implies that $W_{\Delta t} f \leqslant W_{\Delta t} g$ ) provided that conditions (1.11) are met.

We denote by $U^{l}: \mathcal{G}_{\rho} \rightarrow \mathbb{R}$ the discrete approximation at time $l \Delta t$ (see (1.10)) and claim that

$$
\sup _{x \in \mathcal{G}_{\rho}}\left|U^{l}(x)-u^{\epsilon}\left(x, t_{l}\right)\right| \leqslant C l \Delta t L, \quad l=0, \ldots, N .
$$

To see this, we use induction on $l$. The estimate is clearly true for $l=0$. Assuming that (3.6) holds for $l$, we obtain

$$
U^{l}(x) \leqslant u^{\epsilon}\left(x, t_{l}\right)+C l \Delta t L, \quad x \in \mathcal{G}_{\rho},
$$

so that the monotonicity of $W_{\Delta t}$ together with (3.5) imply

$$
\begin{aligned}
U^{l+1}(x)-u^{\epsilon}\left(x, t_{l+1}\right) & =\left(W_{\Delta t} U^{l}\right)(x)-u^{\epsilon}\left(x, t_{l+1}\right) \\
& \leqslant\left(W_{\Delta t}\left(u^{\epsilon}\left(., t_{l}\right)+C l \Delta t L\right)\right)(x)-u^{\epsilon}\left(x, t_{l+1}\right) \\
& =\left(W_{\Delta t} u^{\epsilon}\left(., t_{l}\right)\right)(x)+C l \Delta t L-u^{\epsilon}\left(x, t_{l+1}\right) \\
& \leqslant C(l+1) \Delta t L .
\end{aligned}
$$

Here we also made use of the relation $W_{\Delta t}(f+c)=W_{\Delta t} f+c$ for all constants $c$ which follows immediately from the definition of $W_{\Delta t}$.

In the same way we obtain $u^{\epsilon}\left(x, t_{l+1}\right)-U^{l+1}(x) \leqslant C(l+1) \Delta t L$ so that (3.6) for $l+1$ follows.

The proof of Theorem 1.3 is now completed by combining Theorem 1.2 and (3.6). Since $l \Delta t \leqslant$ $N \Delta t=T$ we obtain

$$
\begin{aligned}
\sup _{x \in \mathcal{G}_{\rho}, l=0, \ldots, N}\left|u\left(x, t_{l}\right)-U^{l}(x)\right| \\
\quad \leqslant \sup _{x \in \mathcal{G}_{\rho}, l=0, \ldots, N}\left|u\left(x, t_{l}\right)-u^{\epsilon}\left(x, t_{l}\right)\right|+\sup _{x \in \mathcal{G}_{\rho}, l=0, \ldots, N}\left|u^{\epsilon}\left(x, t_{l}\right)-U^{l}(x)\right| \\
\quad \leqslant C \epsilon^{\alpha}+C\left(\rho^{2} h^{-2} \epsilon^{-2}+\rho^{2} \epsilon^{-6} h^{-1}+h^{2 \alpha} \epsilon^{-4 \alpha}+\Delta t \epsilon^{-3}+K \rho h^{-1} \epsilon^{-2}\right)
\end{aligned}
$$

provided $h \leqslant \lambda \epsilon^{2}$. We now choose the parameters in such a way that on one hand the conditions (1.11) are satisfied and on the other hand the two terms on the right-hand side of the above inequality balance. This leads to the choices

$$
h=\epsilon^{\frac{5}{2}}, \quad \rho=\epsilon^{\frac{11}{2}}, \quad \Delta t=c_{1} \epsilon^{\frac{17}{2}}, \quad K=c_{2} \epsilon^{-\frac{1}{2}}
$$

which satisfy (1.11) if $c_{1}$ and $c_{2}$ are suitably chosen. In addition we have $h \leqslant \lambda \epsilon^{2}$ for small $\epsilon$. As a result

$$
\sup _{x \in \mathcal{G}_{\rho}, l=0, \ldots, N}\left|u\left(x, t_{l}\right)-U^{l}(x)\right| \leqslant C \epsilon^{\alpha}
$$

and the theorem is proved. 


\section{Estimates for higher order derivatives}

In this section we shall prove the various estimates on the higher order derivatives of $u^{\epsilon}$ which were needed to control the local truncation error of $W_{\Delta t}$. For our approach the following observation made in [10] is crucial: if $u^{\epsilon}$ is a solution of (1.3), then the function $U^{\epsilon}: \mathbb{R}^{n} \times[0, \infty) \rightarrow \mathbb{R}$ given by $U^{\epsilon}(x, t):=\frac{1}{\epsilon} u^{\epsilon}(x, t)$ solves the equation

$$
\begin{aligned}
U_{t}^{\epsilon}=\frac{1}{\epsilon} u_{t}^{\epsilon} & =\left(\delta_{i j}-\frac{u_{x_{i}}^{\epsilon} u_{x_{j}}^{\epsilon}}{\epsilon^{2}+\left|D u^{\epsilon}\right|^{2}}\right) \frac{1}{\epsilon} u_{x_{i} x_{j}}^{\epsilon} \\
& =\left(\delta_{i j}-\frac{U_{x_{i}}^{\epsilon} U_{x_{j}}^{\epsilon}}{1+\left|D U^{\epsilon}\right|^{2}}\right) U_{x_{i} x_{j}}^{\epsilon} \\
& =\sqrt{1+\left|D U^{\epsilon}\right|^{2}} \operatorname{div}\left(\frac{D U^{\epsilon}}{\sqrt{1+\left|D U^{\epsilon}\right|^{2}}}\right) .
\end{aligned}
$$

This means that the graph $\Gamma_{t}^{\epsilon}=\left\{\left(x, x_{n+1}\right) \in \mathbb{R}^{n+1} \mid x_{n+1}=U^{\epsilon}(x, t)\right\}$ of $U^{\epsilon}(., t)$ is moving by mean curvature. Mean curvature evolution of surfaces, which can be written as a graph over some hyperplane, has been studied by Ecker \& Huisken in [8] where the authors examine the existence of global solutions and their asymptotic behavior. In particular, they obtain estimates for the curvature and higher order derivatives of the curvature. It turns out that these estimates can be translated into estimates on derivatives of $u^{\epsilon}$ by exploiting the relation between $\Gamma_{t}^{\epsilon}$ and $u^{\epsilon}$.

Before we carry out this idea, let us introduce some notation from differential geometry. Let $F: V \rightarrow \mathbb{R}^{n+1}\left(V \subset \mathbb{R}^{n}\right.$ open) be a parametrization of a smooth, $n$-dimensional manifold. The induced metric $G=\left(g_{i j}\right)$ and the second fundamental form $A=\left(h_{i j}\right)$ are defined by

$$
g_{i j}=\left\langle\frac{\partial F}{\partial x_{i}}, \frac{\partial F}{\partial x_{j}}\right\rangle, \quad h_{i j}=\left\langle\frac{\partial^{2} F}{\partial x_{i} \partial x_{j}}, N\right\rangle, \quad i, j=1, \ldots, n
$$

where $N$ is the normal to the manifold. We denote by $g^{i j}$ the components of the inverse matrix of $G$. The Christoffel symbols of the second kind are given by

$$
\Gamma_{i j}^{k}=\frac{1}{2} g^{k l}\left(\frac{\partial}{\partial x_{i}} g_{j l}+\frac{\partial}{\partial x_{j}} g_{i l}-\frac{\partial}{\partial x_{l}} g_{i j}\right), \quad i, j, k=1, \ldots, n .
$$

In what follows, we regard all functions, vectors and tensors as being given on $V$. Hence, the covariant derivative of a function $f$ is defined by

$$
\nabla_{j} f=\frac{\partial}{\partial x_{j}} f, \quad j=1, \ldots, n .
$$

The covariant derivative of a covariant vector $X=\left(X_{i}\right)$ is given by

$$
\nabla_{j} X_{i}=\frac{\partial}{\partial x_{j}} X_{i}-\Gamma_{i j}^{k} X^{k}, \quad i, j=1, \ldots, n
$$


and this definition is extended to tensors so as to preserve the product rule and contractions. Thus, for a covariant tensor $T=\left(T_{i k}\right)$ of order 2

$$
\nabla_{j} T_{i k}=\frac{\partial}{\partial x_{j}} T_{i k}-\Gamma_{j k}^{l} T_{i l}-\Gamma_{j i}^{l} T_{k l}, \quad i, j, k=1, \ldots, n .
$$

The Laplace Beltrami operator of a function $f$ is introduced by

$$
\Delta f=g^{i j} \nabla_{i} \nabla_{j} f
$$

Next, the inner product of two covariant vectors $X=\left(X_{i}\right), Y=\left(Y_{i}\right)$ is

$$
X \cdot Y=g^{i j} X_{i} Y_{j},
$$

while the lengths of covariant vectors $\left(X_{i}\right)$ and tensors $\left(T_{i j}\right),\left(S_{i j k}\right)$ are

$$
|X|^{2}=g^{i j} X_{i} X_{j}, \quad|T|^{2}=g^{i j} g^{k l} T_{i k} T_{j l}, \quad|S|^{2}=g^{i j} g^{k l} g^{r s} S_{i k r} S_{j l s} .
$$

Let us now compute the above expressions for the manifold $\Gamma_{t}^{\epsilon}$. Since $\Gamma_{t}^{\epsilon}$ is parametrized by $F^{\epsilon}(., t): \mathbb{R}^{n} \rightarrow \mathbb{R}^{n+1}, F^{\epsilon}(x, t):=\left(x, U^{\epsilon}(x, t)\right)=\left(x, \frac{1}{\epsilon} u^{\epsilon}(x, t)\right)$ we obtain

$$
g_{i j}=\left\langle\frac{\partial F^{\epsilon}}{\partial x_{i}}, \frac{\partial F^{\epsilon}}{\partial x_{j}}\right\rangle=\delta_{i j}+\frac{1}{\epsilon^{2}} u_{x_{i}}^{\epsilon} u_{x_{j}}^{\epsilon}, \quad i, j=1, \ldots, n .
$$

Thus, $G=I+\frac{1}{\epsilon^{2}} D u^{\epsilon} \otimes D u^{\epsilon}$ and therefore

$$
\left(g^{i j}\right)=G^{-1}=I-\frac{1}{\epsilon^{2}+\left|D u^{\epsilon}\right|^{2}} D u^{\epsilon} \otimes D u^{\epsilon} .
$$

Since the normal $N^{\epsilon}$ is given by $N^{\epsilon}=\frac{1}{\sqrt{\epsilon^{2}+\left|D u^{\epsilon}\right|^{2}}}\left(-D u^{\epsilon}, \epsilon\right)$ we compute for the second fundamental form

$$
h_{i j}=\left\langle\frac{\partial^{2} F^{\epsilon}}{\partial x_{i} \partial x_{j}}, N^{\epsilon}\right\rangle=\frac{u_{x_{i} x_{j}}^{\epsilon}}{\sqrt{\epsilon^{2}+\left|D u^{\epsilon}\right|^{2}}}, \quad i, j=1, \ldots, n .
$$

In particular, we have for the norm $|A|$ of the second fundamental form

$$
|A|^{2}=g^{i j} g^{k l} h_{i k} h_{j l}=g^{i j} g^{k l} \frac{u_{x_{i} x_{k}}^{\epsilon} u_{x_{j} x_{l}}^{\epsilon}}{\epsilon^{2}+\left|D u^{\epsilon}\right|^{2}} .
$$

This relation already indicates that bounds on second derivatives of $u^{\epsilon}$ can be derived from estimates on $|A|$. In order to apply a corresponding result from [8] we introduce

$$
v:=\left\langle N^{\epsilon}, e_{n+1}\right\rangle^{-1}=\frac{1}{\epsilon} \sqrt{\epsilon^{2}+\left|D u^{\epsilon}\right|^{2}} .
$$


From [8], Lemma 4.1, and [16], Corollary 3.5, we have the following evolution equations

$$
\begin{aligned}
\frac{\partial}{\partial t} v^{2}-\Delta v^{2} & =-2|A|^{2} v^{2}-6|\nabla v|^{2} \\
\frac{\partial}{\partial t}|A|^{2}-\Delta|A|^{2} & =-2|\nabla A|^{2}+2|A|^{4} .
\end{aligned}
$$

Note that according to (4.3)

$$
|\nabla v|^{2}=g^{i j} v_{x_{i}} v_{x_{j}}, \quad|\nabla A|^{2}=g^{i j} g^{k l} g^{r s} \nabla_{i} h_{k r} \nabla_{j} h_{l s} .
$$

The relations (4.6) and (4.7) are the main tool to prove the following curvature bound:

Lemma 4.1 The second fundamental form of $\Gamma_{t}^{\epsilon}$ satisfies

$$
\sup _{\Gamma_{t}^{\epsilon}}|A|^{2} v^{2} \leqslant \sup _{\Gamma_{0}^{\epsilon}}|A|^{2} v^{2}, \quad t \geqslant 0 .
$$

Proof. Corollary 4.2 in [8].

Lemma 4.1 provides us with a bound on the second derivatives of $u^{\epsilon}$ :

COROLLARY 4.2 The solution $u^{\epsilon}$ of (1.3), (1.4) satisfies

$$
\left|D^{2} u^{\epsilon}(x, t)\right| \leqslant C \epsilon^{-2}\left(\epsilon^{2}+\left|D u^{\epsilon}(x, t)\right|^{2}\right) \quad(x, t) \in \mathbb{R}^{n} \times[0, \infty) .
$$

In particular, we have for $t \geqslant 0$

$$
\left\|D^{2} u^{\epsilon}(., t)\right\|_{L^{\infty}} \leqslant C \epsilon^{-2} .
$$

Proof. Lemma 4.1, (4.4) and the definition of $v$ give for $t \geqslant 0$

$$
\begin{aligned}
\epsilon^{-2} \sup _{x \in \mathbb{R}^{n}}\left(g^{i j} g^{k l} u_{x_{i} x_{k}}^{\epsilon} u_{x_{j} x_{l}}^{\epsilon}\right)(x, t) & =\sup _{\Gamma_{t}^{\epsilon}} v^{2}|A|^{2} \\
& \leqslant \sup _{\Gamma_{0}^{\epsilon}} v^{2}|A|^{2} \\
& =\epsilon^{-2} \sup _{x \in \mathbb{R}^{n}}\left(g^{i j} g^{k l} u_{x_{i} x_{k}}^{\epsilon} u_{x_{j} x_{l}}^{\epsilon}\right)(x, 0) \\
& \leqslant C \epsilon^{-2} \sup _{x \in \mathbb{R}^{n}}\left|D^{2} u_{0}(x)\right|^{2} .
\end{aligned}
$$

Thus, our assumption (2.1) on $u_{0}$ implies

$$
\sup _{x \in \mathbb{R}^{n}}\left(g^{i j} g^{k l} u_{x_{i} x_{k}}^{\epsilon} u_{x_{j} x_{l}}^{\epsilon}\right)(x, t) \leqslant C .
$$

Let us now fix $(x, t) \in \mathbb{R}^{n} \times[0, \infty)$. If $D u^{\epsilon}(x, t)=0$, then $G(x, t)=I$, so that

$$
\left|D^{2} u^{\epsilon}(x, t)\right|^{2}=\left(g^{i j} g^{k l} u_{x_{i} x_{k}}^{\epsilon} u_{x_{j} x_{l}}^{\epsilon}\right)(x, t) \leqslant C
$$


by (4.9), which proves (4.8) for this case.

If $D u^{\epsilon}(x, t) \neq 0$, we let $v_{1}:=\frac{D u^{\epsilon}(x, t)}{\left|D u^{\epsilon}(x, t)\right|}$ and choose $v_{2}, \ldots, v_{n}$ such that $\left\{v_{1}, \ldots, v_{n}\right\}$ is an orthonormal basis for $\mathbb{R}^{n}$. Since

$$
g^{i j}(x, t)=\delta_{i j}-v_{1, i} v_{1, j}+\frac{\epsilon^{2}}{\epsilon^{2}+\left|D u^{\epsilon}\right|^{2}} v_{1, i} v_{1, j}
$$

a short calculation shows

$$
\begin{aligned}
g^{i j} g^{k l} u_{x_{i} x_{k}}^{\epsilon} & u_{x_{j} x_{l}}^{\epsilon} \\
& =\sum_{i, j=2}^{n}\left(u_{v_{i} v_{j}}^{\epsilon}\right)^{2}+\frac{2 \epsilon^{2}}{\epsilon^{2}+\left|D u^{\epsilon}\right|^{2}} \sum_{i=2}^{n}\left(u_{v_{i} v_{1}}^{\epsilon}\right)^{2}+\frac{\epsilon^{4}}{\left(\epsilon^{2}+\left|D u^{\epsilon}\right|^{2}\right)^{2}}\left(u_{v_{1} v_{1}}^{\epsilon}\right)^{2} \\
& \geqslant \frac{\epsilon^{4}}{\left(\epsilon^{2}+\left|D u^{\epsilon}\right|^{2}\right)^{2}}\left|D^{2} u^{\epsilon}\right|^{2}
\end{aligned}
$$

all functions being evaluated at $(x, t)$. Combining this estimate with (4.9) proves (4.8). The second estimate then follows from (1.5).

Bounds on third derivatives of $u^{\epsilon}$ are now obtained in an analogous way, namely by estimating the first derivatives of $A$.

LEMMA 4.3 There exists a constant $C=C\left(u_{0}, T\right)$ such that

$$
\sup _{\Gamma_{t}^{\epsilon}} v^{2}|\nabla A|^{2} \leqslant C \epsilon^{-6}, \quad 0 \leqslant t \leqslant T
$$

Proof. We argue along the lines of Lemma 4.1 in [8] and calculate

$$
\begin{aligned}
& \frac{\partial}{\partial t}\left(v^{2}|\nabla A|^{2}\right)-\Delta\left(v^{2}|\nabla A|^{2}\right) \\
& \quad=v^{2}\left(\frac{\partial}{\partial t}|\nabla A|^{2}-\Delta|\nabla A|^{2}\right)+|\nabla A|^{2}\left(\frac{\partial}{\partial t} v^{2}-\Delta v^{2}\right)-2 \nabla v^{2} \cdot \nabla|\nabla A|^{2} .
\end{aligned}
$$

Proposition 2.3 in [16] gives

$$
\frac{\partial}{\partial t}|\nabla A|^{2}-\Delta|\nabla A|^{2} \leqslant-2\left|\nabla^{2} A\right|^{2}+C|A|^{2}|\nabla A|^{2}
$$

which combined with (4.6) implies

$$
\begin{aligned}
& \frac{\partial}{\partial t}\left(v^{2}|\nabla A|^{2}\right)-\Delta\left(v^{2}|\nabla A|^{2}\right) \\
& \leqslant-2 v^{2}\left|\nabla^{2} A\right|^{2}+C v^{2}|A|^{2}|\nabla A|^{2}-2 v^{2}|A|^{2}|\nabla A|^{2} \\
& \quad-6|\nabla A|^{2}|\nabla v|^{2}-2 \nabla v^{2} \cdot \nabla|\nabla A|^{2}
\end{aligned}
$$


Similarly as in the proof of Lemma 4.1 in [8] we obtain with the help of Young's inequality

$$
\begin{aligned}
-2 \nabla v^{2} \cdot \nabla|\nabla A|^{2}=-\nabla v^{2} \cdot \nabla|\nabla A|^{2}-4 v|\nabla A| \nabla v \cdot \nabla|\nabla A| \\
\leqslant-v^{-2} \nabla v^{2} \cdot \nabla\left(v^{2}|\nabla A|^{2}\right)+v^{-2}\left|\nabla v^{2}\right|^{2}|\nabla A|^{2}+4 v|\nabla A|\left|\nabla^{2} A\right||\nabla v| \\
\quad=-2 v^{-1} \nabla v \cdot \nabla\left(v^{2}|\nabla A|^{2}\right)+6|\nabla v|^{2}|\nabla A|^{2}+2 v^{2}\left|\nabla^{2} A\right|^{2} .
\end{aligned}
$$

Inserting this estimate into (4.11) and using Lemma 4.1 we arrive at

$$
\begin{aligned}
\frac{\partial}{\partial t}\left(v^{2}|\nabla A|^{2}\right) & -\Delta\left(v^{2}|\nabla A|^{2}\right) \\
\leqslant & -2 v^{-1} \nabla v \cdot \nabla\left(v^{2}|\nabla A|^{2}\right)+C v^{2}|A|^{2}|\nabla A|^{2} \\
\leqslant & -2 v^{-1} \nabla v \cdot \nabla\left(v^{2}|\nabla A|^{2}\right)+C \epsilon^{-2}|\nabla A|^{2} .
\end{aligned}
$$

Before we deal with the right-hand side of this estimate we derive an inequality which will be useful in subsequent calculations. From (4.5) we obtain $\nabla_{i} v=-v^{2}\left\langle\nabla_{i} N^{\epsilon}, e_{n+1}\right\rangle$ which implies

$$
|\nabla v| \leqslant v^{2}\left|\nabla N^{\epsilon}\right|=v^{2}|A|
$$

by (4.22) below. Let us return to (4.12) and use (4.7) and (4.13) to obtain

$$
\begin{aligned}
\frac{\partial}{\partial t}|A|^{2}-\Delta|A|^{2} & +2 v^{-1} \nabla v \cdot \nabla|A|^{2} \\
& \leqslant-2|\nabla A|^{2}+2|A|^{4}+4 v^{-1}|\nabla v||A||\nabla A| \\
& \leqslant-2|\nabla A|^{2}+2|A|^{4}+4 v|A|^{2}|\nabla A| \\
& \leqslant-|\nabla A|^{2}+2|A|^{4}+4 v^{2}|A|^{4} \\
& \leqslant-|\nabla A|^{2}+C \epsilon^{-4}
\end{aligned}
$$

where we used Hölder's inequality together with the fact that $|A|^{2} \leqslant C \epsilon^{-2}$ and $|A|^{2} v^{2} \leqslant C \epsilon^{-2}$ by Lemma 4.1. Thus, if we choose $\beta>C$, (4.12) and (4.14) give

$$
\begin{aligned}
\frac{\partial}{\partial t}\left(v^{2}|\nabla A|^{2}\right. & \left.+\frac{\beta}{\epsilon^{2}}|A|^{2}\right)-\Delta\left(v^{2}|\nabla A|^{2}+\frac{\beta}{\epsilon^{2}}|A|^{2}\right) \\
\leqslant & -2 v^{-1} \nabla v \cdot \nabla\left(v^{2}|\nabla A|^{2}+\frac{\beta}{\epsilon^{2}}|A|^{2}\right)+C \epsilon^{-6} .
\end{aligned}
$$

As a consequence, the function $f:=v^{2}|\nabla A|^{2}+\frac{\beta}{\epsilon^{2}}|A|^{2}-C \epsilon^{-6} t$ satisfies

$$
\frac{\partial f}{\partial t}-\Delta f \leqslant-2 v^{-1} \nabla v \cdot \nabla f .
$$

Corollary 1.1 in [8] then implies $\sup f \leqslant \sup f$ for $t \geqslant 0$ so that

$$
\sup _{\Gamma_{t}^{\epsilon}} v^{2}|\nabla A|^{2} \leqslant C \epsilon^{-6}+\sup _{\Gamma_{0}^{\epsilon}} v^{2}|\nabla A|^{2}+\frac{\beta}{\epsilon^{2}} \sup _{\Gamma_{0}^{\epsilon}}|A|^{2} .
$$


In order to estimate $v^{2}|\nabla A|^{2}$ at $t=0$ we compute the covariant derivative of the second fundamental form. According to (4.2)

$$
\nabla_{i} h_{k r}=\frac{\partial}{\partial x_{i}} h_{k r}-\Gamma_{i k}^{m} h_{m r}-\Gamma_{i r}^{m} h_{m k}
$$

and a short calculation shows that the Christoffel symbols are given by

$$
\Gamma_{i k}^{m}=\frac{u_{x_{m}}^{\epsilon} u_{x_{i} x_{k}}^{\epsilon}}{\epsilon^{2}+\left|D u^{\epsilon}\right|^{2}}, \quad i, k, m=1, \ldots, n .
$$

Thus,

$$
\begin{aligned}
\nabla_{i} h_{k r} & =\frac{\partial}{\partial x_{i}}\left(\frac{u_{x_{k} x_{r}}^{\epsilon}}{\sqrt{\epsilon^{2}+\left|D u^{\epsilon}\right|^{2}}}\right)-\frac{u_{x_{m}}^{\epsilon} u_{x_{i} x_{k}}^{\epsilon} u_{x_{m} x_{r}}^{\epsilon}}{\sqrt{\epsilon^{2}+\left|D u^{\epsilon}\right|^{2}}}-\frac{u_{x_{m}}^{\epsilon} u_{x_{i} x_{r}}^{\epsilon} u_{x_{m} x_{k}}^{\epsilon}}{\sqrt{\epsilon^{2}+\left|D u^{\epsilon}\right|^{2}}} \\
& =\frac{u_{x_{i} x_{k} x_{r}}}{\sqrt{\epsilon^{2}+\left|D u^{\epsilon}\right|^{2}}}-\frac{\epsilon}{\sqrt{\epsilon^{2}+\left|D u^{\epsilon}\right|^{2}}}\left(h_{k r} \nabla_{i} v+h_{i k} \nabla_{r} v+h_{i r} \nabla_{k} v\right) .
\end{aligned}
$$

Taking into account that $g^{i j}=\delta_{i j}-\frac{u_{x_{i}}^{\epsilon} u_{x_{j}}^{\epsilon}}{\epsilon^{2}+\left|D u^{\epsilon}\right|^{2}}$, from (2.1) and (4.13) we obtain

$$
\sup _{\Gamma_{0}^{\epsilon}} v^{2}|\nabla A|^{2} \leqslant \frac{C}{\epsilon^{2}} \sup _{\mathbb{R}^{n}}\left|D^{3} u_{0}\right|^{2}+C \sup _{\Gamma_{0}^{\epsilon}}|A|^{2}|\nabla v|^{2} \leqslant \frac{C}{\epsilon^{2}}+C \sup _{\Gamma_{0}^{\epsilon}} v^{4}|A|^{4} \leqslant C \epsilon^{-4} .
$$

Inserting this estimate into (4.16) finally proves the lemma.

Lemma 4.3 now allows us to bound the third derivatives of $u^{\epsilon}$.

Lemma 4.4 The solution $u^{\epsilon}$ of (1.3), (1.4) satisfies

$$
\left\|D^{3} u^{\epsilon}(., t)\right\|_{L^{\infty}} \leqslant C \epsilon^{-5}, \quad 0 \leqslant t \leqslant T .
$$

Proof. To begin, (4.17) immediately implies

$$
\begin{aligned}
g^{i j} g^{k l} g^{r s} u_{x_{i} x_{k} x_{r}}^{\epsilon} u_{x_{j} x_{l} x_{s}}^{\epsilon} & \leqslant\left(\epsilon^{2}+\left|D u^{\epsilon}\right|^{2}\right)|\nabla A|^{2}+C \epsilon^{2}|A|^{2}|\nabla v|^{2} \\
& \leqslant \epsilon^{2} v^{2}|\nabla A|^{2}+C \epsilon^{2}|A|^{4} v^{4} \\
& \leqslant C \epsilon^{-4}
\end{aligned}
$$

by (4.13) and Lemma 4.3. On the other hand, a similar calculation as in the proof of Corollary 4.2 shows

$$
C \epsilon^{-4} \geqslant g^{i j} g^{k l} g^{r s} u_{x_{i} x_{k} x_{r}}^{\epsilon} u_{x_{j} x_{l} x_{s}}^{\epsilon} \geqslant \frac{\epsilon^{6}}{\left(\epsilon^{2}+\left|D u^{\epsilon}\right|^{2}\right)^{3}}\left|D^{3} u^{\epsilon}\right|^{2} .
$$

The assertion of the lemma now follows from (1.5).

The second derivatives of $A$ can be estimated in a similar way as in Lemma 4.3. We leave the details to the reader and just mention that the function $f$ which appears in (4.15) is now replaced by

$$
g:=v^{2}\left|\nabla^{2} A\right|^{2}+\frac{\gamma_{1}}{\epsilon^{2}}|\nabla A|^{2}+\frac{\gamma_{2}}{\epsilon^{4}}|A|^{2}-C \epsilon^{-8} t .
$$

Thus we obtain 
Lemma 4.5 There exists a constant $C=C\left(u_{0}, T\right)$ such that

$$
\sup _{\Gamma_{t}^{\epsilon}} v^{2}\left|\nabla^{2} A\right|^{2} \leqslant C \epsilon^{-8}, \quad 0 \leqslant t \leqslant T .
$$

As a consequence of the above curvature bounds we can estimate the second time derivative of $u^{\epsilon}$.

COROLlary 4.6 The solution $u^{\epsilon}$ of (1.3), (1.4) satisfies

$$
\sup _{\mathbb{R}^{n}}\left\|u_{t t}^{\epsilon}(., t)\right\|_{L^{\infty}} \leqslant C \epsilon^{-3}, \quad 0 \leqslant t \leqslant T .
$$

Proof. In view of (4.1) we have

$$
u_{t}^{\epsilon}=\epsilon \sqrt{1+\left|D U^{\epsilon}\right|^{2}} H=\sqrt{\epsilon^{2}+\left|D u^{\epsilon}\right|^{2}} H=\epsilon v H
$$

where $H$ is the mean curvature of $\Gamma_{t}^{\epsilon}$. Differentiating this identity with respect to time yields

$$
u_{t t}^{\epsilon}=\epsilon v_{t} H+\epsilon v H_{t} .
$$

Clearly, $\epsilon v_{t}=\frac{u_{x_{i}}^{\epsilon} u_{t, x_{i}}^{\epsilon}}{\sqrt{\epsilon^{2}+\left|D u^{\epsilon}\right|^{2}}}$ and therefore $\epsilon\left|v_{t}\right| \leqslant\left|D u_{t}^{\epsilon}\right|$. We would like to estimate $\left|D u_{t}^{\epsilon}\right|$ by $\left|\nabla u_{t}^{\epsilon}\right|$. To do so, note that

$$
g^{i j} \xi_{i} \xi_{j}=|\xi|^{2}-\frac{\left\langle\xi, D u^{\epsilon}\right\rangle^{2}}{\epsilon^{2}+\left|D u^{\epsilon}\right|^{2}} \geqslant \frac{\epsilon^{2}}{\epsilon^{2}+\left|D u^{\epsilon}\right|^{2}}|\xi|^{2}=v^{-2}|\xi|^{2} \quad \text { for all } \xi \in \mathbb{R}^{n}
$$

so that

$$
\epsilon\left|v_{t}\right| \leqslant\left|D u_{t}^{\epsilon}\right| \leqslant v\left(g^{i j} u_{t, x_{i}}^{\epsilon} u_{t, x_{j}}^{\epsilon}\right)^{\frac{1}{2}}=v\left|\nabla u_{t}^{\epsilon}\right|
$$

Returning to (4.18) we obtain $\nabla_{i} u_{t}^{\epsilon}=\epsilon \nabla_{i} v H+\epsilon v \nabla_{i} H$ which implies

$$
\begin{aligned}
\left|\nabla u_{t}^{\epsilon}\right| & \leqslant \epsilon|\nabla v||H|+\epsilon v|\nabla H| \leqslant \epsilon v^{2}|A||H|+\epsilon v|\nabla A| \\
& =\left|u_{t}^{\epsilon}\right| v|A|+\epsilon v|\nabla A| \leqslant C \epsilon^{-2}
\end{aligned}
$$

by (4.13), (4.1), (1.5), Lemma 4.1 and Lemma 4.3.

According to [15], Corollary 3.5(i), we have $H_{t}=\Delta H+|A|^{2} H$ which combined with (4.20) and (4.21) gives

$$
\begin{aligned}
\left|u_{t t}^{\epsilon}\right| & \leqslant \epsilon\left|v_{t}\right||H|+\epsilon v\left|H_{t}\right| \\
& \leqslant v\left|\nabla u_{t}^{\epsilon}\right||H|+\epsilon v\left(|\Delta H|+|A|^{2}|H|\right) \\
& \leqslant \frac{1}{\epsilon}\left|u_{t}^{\epsilon}\right|\left|\nabla u_{t}^{\epsilon}\right|+\epsilon v\left|\nabla^{2} A\right|+\left|u_{t}^{\epsilon}\right||A|^{2} \\
& \leqslant C \epsilon^{-3}
\end{aligned}
$$

where we made use of (4.1), (1.5), Lemma 4.1 and Lemma 4.5. 
Before we turn to a local Hölder-estimate for the second derivatives of $u^{\epsilon}$ we establish a bound on the derivatives of the the matrix $B(x, t)=\left(b_{i j}(x, t)\right)_{i j}=\left(\theta_{i j}^{\epsilon}\left(D u^{\epsilon}(x, t)\right)\right)_{i j}$.

LEMMA 4.7 We have for all $\epsilon>0$ :

$$
\sup _{1 \leqslant i, j \leqslant n} \sup _{x \in \mathbb{R}^{n}, t \geqslant 0}\left|D b_{i j}(x, t)\right| \leqslant \frac{C}{\epsilon} .
$$

Proof. We start by estimating the covariant derivative of the normal

$$
N^{\epsilon}=\left(\frac{-D u^{\epsilon}}{\sqrt{\epsilon^{2}+\left|D u^{\epsilon}\right|^{2}}}, \frac{\epsilon}{\sqrt{\epsilon^{2}+\left|D u^{\epsilon}\right|^{2}}}\right)=:\left(v^{\epsilon}, \frac{\epsilon}{\sqrt{\epsilon^{2}+\left|D u^{\epsilon}\right|^{2}}}\right) .
$$

Since $\frac{\partial}{\partial x_{i}} N^{\epsilon}=h_{i k} g^{k m} \frac{\partial F^{\epsilon}}{\partial x_{m}}$ we obtain

$$
\left|\nabla N^{\epsilon}\right|^{2}=g^{i j}\left\langle N_{x_{i}}^{\epsilon}, N_{x_{j}}^{\epsilon}\right\rangle=g^{i j} h_{i k} g^{k m} h_{j l} g^{l r}\left\langle\frac{\partial F^{\epsilon}}{\partial x_{m}}, \frac{\partial F^{\epsilon}}{\partial x_{r}}\right\rangle=g^{i j} g^{k l} h_{i k} h_{j l}=|A|^{2}
$$

on $\Gamma_{t}^{\epsilon}$. Furthermore, writing

$$
B=\theta^{\epsilon}\left(D u^{\epsilon}\right)=I-\frac{1}{1+\frac{\epsilon}{\sqrt{\epsilon^{2}+\left|D u^{\epsilon}\right|^{2}}}} \frac{D u^{\epsilon} \otimes D u^{\epsilon}}{\epsilon^{2}+\left|D u^{\epsilon}\right|^{2}}=I-\frac{1}{1+v^{-1}} v^{\epsilon} \otimes v^{\epsilon}
$$

we may estimate, by using (4.13) and (4.22),

$$
\begin{aligned}
\left|\nabla b_{i j}\right| & \leqslant \frac{1}{\left(1+v^{-1}\right)^{2}} \frac{|\nabla v|}{v^{2}}+\frac{2}{1+v^{-1}}\left|\nabla v^{\epsilon}\right| \\
& \leqslant \frac{1}{(v+1)^{2}}|\nabla v|+\frac{2 v}{v+1}\left|\nabla N^{\epsilon}\right| \\
& \leqslant \frac{v^{2}}{(v+1)^{2}}|A|+2|A| \\
& \leqslant 3|A| .
\end{aligned}
$$

Employing (4.19) we finally obtain

$$
\left|D b_{i j}\right| \leqslant v\left|\nabla b_{i j}\right| \leqslant 3 v|A| \leqslant \frac{C}{\epsilon}
$$

by Lemma 4.1.

We are now in position to prove a Hölder-continuity result for the second derivatives of $u^{\epsilon}$.

Lemma 4.8 Let $u^{\epsilon}$ be the solution of (1.3), (1.4) and $\beta \in(0,1)$. There exist $C \geqslant 0$ and $\lambda>0$ which only depend on $u_{0}, \beta, T$ and $n$ such that for all $\left(x_{0}, t_{0}\right) \in \mathbb{R}^{n} \times[0, \infty)$

$$
\sup _{i, j=1, \ldots, n}\left|\left\langle\left(D^{2} u^{\epsilon}\left(x_{0}+P^{\epsilon} y, t_{0}\right)-D^{2} u^{\epsilon}\left(x_{0}, t_{0}\right)\right) P^{\epsilon} e_{i}, P^{\epsilon} e_{j}\right\rangle\right| \leqslant C \epsilon^{-2 \beta}|y|^{\beta}
$$

provided $|y| \leqslant \lambda \epsilon^{2}$. Here, $P^{\epsilon}:=\theta^{\epsilon}\left(D u^{\epsilon}\left(x_{0}, t_{0}\right)\right) \in \mathcal{S}(n)$. 
Proof. Let us define $w^{\epsilon}: \mathbb{R}^{n} \rightarrow \mathbb{R}$ by

$$
w^{\epsilon}(y):=u^{\epsilon}\left(x_{0}+P^{\epsilon} y, t_{0}\right)-u^{\epsilon}\left(x_{0}, t_{0}\right)-\left\langle D u^{\epsilon}\left(x_{0}, t_{0}\right), P^{\epsilon} y\right\rangle .
$$

The derivatives of $w^{\epsilon}$ are

$$
\begin{aligned}
w_{y_{i}}^{\epsilon}(y) & =\left\langle D u^{\epsilon}\left(x_{0}+P^{\epsilon} y, t_{0}\right)-D u^{\epsilon}\left(x_{0}, t_{0}\right), P^{\epsilon} e_{i}\right\rangle \quad i=1, \ldots, n \\
w_{y_{i} y_{j}}^{\epsilon}(y) & =\left\langle D^{2} u^{\epsilon}\left(x_{0}+P^{\epsilon} y, t_{0}\right) P^{\epsilon} e_{i}, P^{\epsilon} e_{j}\right\rangle \quad i, j=1, \ldots, n .
\end{aligned}
$$

Thus, our lemma will be implied by the estimate

$$
\left\|D^{2} w^{\epsilon}\right\|_{C^{0, \beta}\left(\bar{B}_{R}\right)} \leqslant C \epsilon^{-2 \beta}
$$

provided $R=\lambda \epsilon^{2}$ and $\lambda$ is chosen appropriately.

To prove (4.24) we regard $w^{\epsilon}$ as the solution of a Poisson equation and use a Schauder estimate. Abbreviating $Q^{\epsilon}(y)=\theta^{\epsilon}\left(D u^{\epsilon}\left(x_{0}+P^{\epsilon} y, t_{0}\right)\right)$ a short calculation shows

$$
\begin{aligned}
\Delta w^{\epsilon}(y)= & \operatorname{trace}\left(D^{2} w^{\epsilon}(y)\right)=\operatorname{trace}\left(P^{\epsilon} D^{2} u^{\epsilon}\left(x_{0}+P^{\epsilon} y, t_{0}\right) P^{\epsilon}\right) \\
= & \operatorname{trace}\left(Q^{\epsilon}(y) D^{2} u^{\epsilon}\left(x_{0}+P^{\epsilon} y, t_{0}\right) Q^{\epsilon}(y)\right) \\
& +\operatorname{trace}\left(D^{2} w^{\epsilon}(y)-S^{\epsilon}(y) D^{2} w^{\epsilon}(y) S^{\epsilon}(y)^{t}\right)
\end{aligned}
$$

where $S^{\epsilon}(y)=Q^{\epsilon}(y)\left(P^{\epsilon}\right)^{-1}=Q^{\epsilon}(y)\left(Q^{\epsilon}(0)\right)^{-1}$. Let $x_{1}:=x_{0}+P^{\epsilon} y$. Remembering the definition of $Q^{\epsilon}$ and (3.3) we obtain

$$
\operatorname{trace}\left(Q^{\epsilon}(y) D^{2} u^{\epsilon}\left(x_{1}, t_{0}\right) Q^{\epsilon}(y)\right)=\sum_{j=1}^{n}\left\langle D^{2} u^{\epsilon}\left(x_{1}, t_{0}\right) Q^{\epsilon}(y) e_{j}, Q^{\epsilon}(y) e_{j}\right\rangle=u_{t}^{\epsilon}\left(x_{1}, t_{0}\right) .
$$

As a result,

$$
\Delta w^{\epsilon}(y)=u_{t}^{\epsilon}\left(x_{0}+P^{\epsilon} y, t_{0}\right)+\operatorname{trace}\left(D^{2} w^{\epsilon}(y)-S^{\epsilon}(y) D^{2} w^{\epsilon}(y) S^{\epsilon}(y)^{t}\right) .
$$

Next, let $\zeta \in C^{3}\left(\mathbb{R}^{n}\right)$ be a cut-off function, $0 \leqslant \zeta \leqslant 1$, supp $\zeta \subset B_{2 R}=B_{2 R}(0), R \ll 1, \zeta \equiv 1$ on $B_{R},\left|D^{l} \zeta\right| \leqslant C R^{-l}, 1 \leqslant l \leqslant 3$ and set $z^{\epsilon}(y):=\zeta(y) w^{\epsilon}(y)$. Then

$$
\begin{array}{rlrl}
\Delta z^{\epsilon} & =F^{\epsilon} & & \text { in } B_{1} \\
z^{\epsilon}=0 & & \text { on } \partial B_{1}
\end{array}
$$

where

$$
\begin{aligned}
F^{\epsilon}(y)= & \zeta(y) u_{t}^{\epsilon}\left(x_{0}+P^{\epsilon} y, t_{0}\right)+\operatorname{trace}\left(D^{2} z^{\epsilon}(y)-S^{\epsilon}(y) D^{2} z^{\epsilon}(y) S^{\epsilon}(y)^{t}\right) \\
& +\operatorname{trace}\left(S^{\epsilon}(y)\left(D w^{\epsilon}(y) \otimes D \zeta(y)+D \zeta(y) \otimes D w^{\epsilon}(y)+w^{\epsilon}(y) D^{2} \zeta(y)\right) S^{\epsilon}(y)^{t}\right) .
\end{aligned}
$$

A well-known Schauder estimate (see [13], Theorem 6.6) implies

$$
\left\|D^{2} z^{\epsilon}\right\|_{C^{0, \beta}\left(\bar{B}_{1}\right)} \leqslant C(\beta, n)\left\|F^{\epsilon}\right\|_{C^{0, \beta}\left(\bar{B}_{1}\right)} .
$$


Before we start to estimate the right-hand side of (4.25) we derive two useful bounds on $I-S^{\epsilon}$.

Note that by (4.23)

$$
P^{\epsilon}=I-\frac{1}{1+v^{-1}} v^{\epsilon} \otimes v^{\epsilon}
$$

so that

$$
\left(P^{\epsilon}\right)^{-1}=I+\frac{v}{1+v^{-1}} v^{\epsilon} \otimes v^{\epsilon}
$$

all functions being evaluated at $\left(x_{0}, t_{0}\right)$. The above identity together with Lemma 4.7 implies

$$
\begin{aligned}
\left|I-S^{\epsilon}(y)\right| & =\left|\left(Q^{\epsilon}(0)-Q^{\epsilon}(y)\right)\left(P^{\epsilon}\right)^{-1}\right| \\
& =\left|\left(\theta^{\epsilon}\left(D u^{\epsilon}\left(x_{0}, t_{0}\right)\right)-\theta^{\epsilon}\left(D u^{\epsilon}\left(x_{0}+P^{\epsilon} y, t_{0}\right)\right)\right)\left(P^{\epsilon}\right)^{-1}\right| \\
& \leqslant \frac{C}{\epsilon}\left|P^{\epsilon} y\right|\left|\left(P^{\epsilon}\right)^{-1}\right| \leqslant \frac{C}{\epsilon} v|y| \leqslant \frac{C}{\epsilon^{2}} R
\end{aligned}
$$

for $y \in B_{2 R}$. Thus, assuming that $R=\lambda \epsilon^{2}$ we arrive at

$$
\left\|I-S^{\epsilon}\right\|_{L^{\infty}\left(B_{2 R}\right)} \leqslant C \lambda \text {. }
$$

Similarly we obtain

$$
\left\|I-S^{\epsilon}\right\|_{C^{0, \beta}\left(\bar{B}_{2 R}\right)} \leqslant C \lambda R^{-\beta} .
$$

A first application of the above estimates are bounds on the function $w^{\epsilon}$. We claim that

$$
\left|w^{\epsilon}(y)\right| \leqslant C R^{2}, \quad\left|D w^{\epsilon}(y)\right| \leqslant C R, \quad\left|D^{2} w^{\epsilon}(y)\right| \leqslant C, \quad|y| \leqslant 2 R .
$$

To prove (4.28) we first establish the bound on the second derivatives. Let us write

$$
\begin{aligned}
D^{2} w^{\epsilon}(y)= & P^{\epsilon} D^{2} u^{\epsilon}\left(x_{0}+P^{\epsilon} y, t_{0}\right) P^{\epsilon} \\
= & Q^{\epsilon}(y) D^{2} u^{\epsilon}\left(x_{0}+P^{\epsilon} y, t_{0}\right) Q^{\epsilon}(y)+D^{2} w^{\epsilon}(y)\left(I-S^{\epsilon}(y)^{t}\right) \\
& \quad+\left(I-S^{\epsilon}(y)\right) D^{2} w^{\epsilon}(y) S^{\epsilon}(y)^{t}
\end{aligned}
$$

so that (4.26) implies

$$
\left|D^{2} w^{\epsilon}(y)\right| \leqslant\left|Q^{\epsilon}(y) D^{2} u^{\epsilon}\left(x_{0}+P^{\epsilon} y, t_{0}\right) Q^{\epsilon}(y)\right|+C \lambda\left|D^{2} w^{\epsilon}(y)\right|, \quad|y| \leqslant 2 R
$$

and therefore

$$
\left|D^{2} w^{\epsilon}(y)\right| \leqslant 2\left|Q^{\epsilon}(y) D^{2} u^{\epsilon}\left(x_{0}+P^{\epsilon} y, t_{0}\right) Q^{\epsilon}(y)\right|
$$

provided $\lambda$ is sufficiently small. In order to estimate the right-hand side we let $x_{1}=x_{0}+P^{\epsilon} y$ and distinguish two cases: if $D u^{\epsilon}\left(x_{1}, t_{0}\right)=0$, then $Q^{\epsilon}(y)=I$ and (4.8) gives

$$
\left|D^{2} w^{\epsilon}(y)\right| \leqslant 2\left|D^{2} u^{\epsilon}\left(x_{0}+P^{\epsilon} y, t_{0}\right)\right| \leqslant C \frac{\epsilon^{2}+\left|D u^{\epsilon}\left(x_{1}, t_{0}\right)\right|^{2}}{\epsilon^{2}}=C .
$$


If $D u^{\epsilon}\left(x_{1}, t_{0}\right) \neq 0$, we introduce an orthonormal basis $\left\{v_{1}, \ldots, v_{n}\right\}$ as in Corollary 4.2 where $v_{1}=\frac{D u^{\epsilon}\left(x_{1}, t_{0}\right)}{\left|D u^{\epsilon}\left(x_{1}, t_{0}\right)\right|}$. In this case we can rewrite $\theta^{\epsilon}\left(D u^{\epsilon}\right)$ as follows:

$$
\begin{aligned}
\theta^{\epsilon}\left(D u^{\epsilon}\right) & =I-\frac{1}{1+\frac{\epsilon}{\sqrt{\epsilon^{2}+\left|D u^{\epsilon}\right|^{2}}}} \frac{D u^{\epsilon} \otimes D u^{\epsilon}}{\epsilon^{2}+\left|D u^{\epsilon}\right|^{2}} \\
& =I-\frac{D u^{\epsilon}}{\left|D u^{\epsilon}\right|} \otimes \frac{D u^{\epsilon}}{\left|D u^{\epsilon}\right|}+\left(1-\frac{1}{1+\frac{\epsilon}{\sqrt{\epsilon^{2}+\left|D u^{\epsilon}\right|^{2}}}} \frac{\left|D u^{\epsilon}\right|^{2}}{\epsilon^{2}+\left|D u^{\epsilon}\right|^{2}}\right) \frac{D u^{\epsilon}}{\left|D u^{\epsilon}\right|} \otimes \frac{D u^{\epsilon}}{\left|D u^{\epsilon}\right|} \\
& =I-\frac{D u^{\epsilon}}{\left|D u^{\epsilon}\right|} \otimes \frac{D u^{\epsilon}}{\left|D u^{\epsilon}\right|}+\frac{\epsilon}{\sqrt{\epsilon^{2}+\left|D u^{\epsilon}\right|^{2}}} \frac{D u^{\epsilon}}{\left|D u^{\epsilon}\right|} \otimes \frac{D u^{\epsilon}}{\left|D u^{\epsilon}\right|} .
\end{aligned}
$$

Thus,

$$
Q^{\epsilon}(y)=I-v_{1} \otimes v_{1}+\frac{\epsilon}{\sqrt{\epsilon^{2}+\left|D u^{\epsilon}\left(x_{1}, t_{0}\right)\right|^{2}}} v_{1} \otimes v_{1}
$$

and a short calculation shows

$$
\begin{aligned}
& Q^{\epsilon}(y) D^{2} u^{\epsilon}\left(x_{0}+P^{\epsilon} y, t_{0}\right) Q^{\epsilon}(y) \\
& =\left(\sum_{k, l=2}^{n} u_{v_{k} v_{l}}^{\epsilon} v_{k, i} v_{l, j}+\frac{\epsilon}{\sqrt{\epsilon^{2}+\left|D u^{\epsilon}\right|^{2}}} \sum_{k=2}^{n} u_{v_{1} v_{k}}^{\epsilon}\left(v_{k, i} v_{1, j}+v_{k, j} v_{1, i}\right)\right. \\
& \left.\quad+\frac{\epsilon^{2}}{\epsilon^{2}+\left|D u^{\epsilon}\right|^{2}} u_{v_{1} v_{1}}^{\epsilon} v_{1, i} v_{1, j}\right)_{i, j} .
\end{aligned}
$$

From (4.9) and (4.10) we deduce that also in this case

$$
\begin{aligned}
\mid Q^{\epsilon}(y) D^{2} u^{\epsilon} & \left(x_{0}+P^{\epsilon} y, t_{0}\right) Q^{\epsilon}(y) \mid \\
& \leqslant C\left(\sum_{k, l=2}^{n}\left|u_{v_{k} v_{l}}^{\epsilon}\right|+\frac{\epsilon}{\sqrt{\epsilon^{2}+\left|D u^{\epsilon}\right|^{2}}} \sum_{k=2}^{n}\left|u_{v_{1} v_{k}}^{\epsilon}\right|+\frac{\epsilon^{2}}{\epsilon^{2}+\left|D u^{\epsilon}\right|^{2}}\left|u_{v_{1} v_{1}}^{\epsilon}\right|\right) \\
& \leqslant C\left(g^{i j} g^{k l} u_{x_{i} x_{k}}^{\epsilon} u_{x_{j} x_{l}}^{\epsilon}\right)^{\frac{1}{2}} \\
& \leqslant C
\end{aligned}
$$

where $u^{\epsilon}$ is evaluated at $\left(x_{1}, t_{0}\right)$. Inserting this estimate into (4.29) gives

$$
\left|D^{2} w^{\epsilon}(y)\right| \leqslant C, \quad|y| \leqslant 2 R .
$$

Furthermore, the remaining estimates in (4.28) follow from the fact that $w^{\epsilon}(0)=0$ and $D w^{\epsilon}(0)=$ 0 . We can now start to estimate $\left\|F^{\epsilon}\right\|_{C^{0, \beta}\left(\bar{B}_{1}\right)}$. In view of the elementary inequality $\|f g\|_{C^{0, \beta}} \leqslant$ $\|f\|_{C^{0, \beta}}\|g\|_{L^{\infty}}+\|f\|_{L^{\infty}}\|g\|_{C^{0, \beta}}$, the properties of $\zeta$ and (4.26)-(4.28) we obtain

$$
\left\|S^{\epsilon}\left(D w^{\epsilon} \otimes D \zeta+D \zeta \otimes D w^{\epsilon}+w^{\epsilon} D^{2} \zeta\right)\left(S^{\epsilon}\right)^{t}\right\|_{C^{0, \beta}\left(\bar{B}_{1}\right)} \leqslant C\left(1+R^{-\beta}\right) .
$$


Next, writing

$$
D^{2} z^{\epsilon}-S^{\epsilon} D^{2} z^{\epsilon}\left(S^{\epsilon}\right)^{t}=\left(I-S^{\epsilon}\right) D^{2} z^{\epsilon}+S^{\epsilon} D^{2} z^{\epsilon}\left(I-\left(S^{\epsilon}\right)^{t}\right)
$$

and observing that $D^{2} z^{\epsilon}(y)=0$ for $|y| \geqslant 2 R$ we obtain

$$
\begin{aligned}
& \left\|D^{2} z^{\epsilon}-S^{\epsilon} D^{2} z^{\epsilon}\left(S^{\epsilon}\right)^{t}\right\|_{C^{0, \beta}\left(\bar{B}_{1}\right)} \\
& \leqslant\left(1+\left\|S^{\epsilon}\right\|_{L^{\infty}\left(B_{2 R}\right)}\right)\left(\left\|I-S^{\epsilon}\right\|_{L^{\infty}\left(B_{2 R}\right)}\left\|D^{2} z^{\epsilon}\right\|_{C^{0, \beta}\left(\bar{B}_{1}\right)}\right. \\
& \left.\quad+\left\|I-S^{\epsilon}\right\|_{C^{0, \beta}\left(\bar{B}_{2 R}\right)}\left\|D^{2} z^{\epsilon}\right\|_{L^{\infty}\left(B_{2 R}\right)}\right) \\
& \leqslant C \lambda\left\|D^{2} z^{\epsilon}\right\|_{C^{0, \beta}\left(\bar{B}_{1}\right)}+C R^{-\beta}\left\|D^{2} z^{\epsilon}\right\|_{L^{\infty}\left(B_{2 R}\right)}
\end{aligned}
$$

by (4.26) and (4.27). The last term on the right-hand side can be estimated further with the help of (4.28) and the properties of the cut-off function, namely

$$
\begin{aligned}
& \left\|D^{2} z^{\epsilon}\right\|_{L^{\infty}\left(B_{2 R}\right)} \\
& \quad \leqslant C\left(\left\|D^{2} w^{\epsilon}\right\|_{L^{\infty}\left(B_{2 R}\right)}+\|D \zeta\|_{L^{\infty}\left(B_{2 R}\right)}\left\|D w^{\epsilon}\right\|_{L^{\infty}\left(B_{2 R}\right)}+\left\|w^{\epsilon}\right\|_{L^{\infty}\left(B_{2 R}\right)}\left\|D^{2} \zeta\right\|_{L^{\infty}\left(B_{2 R}\right)}\right) \\
& \quad \leqslant C .
\end{aligned}
$$

If we insert this inequality into (4.31), the result is

$$
\left\|D^{2} z^{\epsilon}-S^{\epsilon} D^{2} z^{\epsilon}\left(S^{\epsilon}\right)^{t}\right\|_{C^{0, \beta}\left(\bar{B}_{1}\right)} \leqslant C \lambda\left\|D^{2} z^{\epsilon}\right\|_{C^{0, \beta}\left(\bar{B}_{1}\right)}+C R^{-\beta} .
$$

Finally, it remains to estimate the Hölder-norm of $\zeta u_{t}^{\epsilon}\left(x_{0}+P^{\epsilon}\right.$., $\left.t_{0}\right)$ on $B_{1}$. Let us define $h^{\epsilon}(y):=$ $u_{t}^{\epsilon}\left(x_{0}+P^{\epsilon} y, t_{0}\right)$ and proceeding in a similar way as above we obtain

$$
\begin{aligned}
\left|D h^{\epsilon}(y)\right|^{2} & =\left\langle D h^{\epsilon}(y), D h^{\epsilon}(y)\right\rangle=\left\langle P^{\epsilon} D u_{t}^{\epsilon}\left(x_{0}+P^{\epsilon} y, t_{0}\right), P^{\epsilon} D u_{t}^{\epsilon}\left(x_{0}+P^{\epsilon} y, t_{0}\right)\right\rangle \\
& \leqslant\left\langle Q^{\epsilon}(y) D u_{t}^{\epsilon}\left(x_{0}+P^{\epsilon} y, t_{0}\right), Q^{\epsilon}(y) D u_{t}^{\epsilon}\left(x_{0}+P^{\epsilon} y, t_{0}\right)\right\rangle+C \lambda\left|D h^{\epsilon}(y)\right|^{2} \\
& =g^{i j}\left(x_{0}+P^{\epsilon} y, t_{0}\right) u_{t, x_{i}}^{\epsilon}\left(x_{0}+P^{\epsilon} y, t_{0}\right) u_{t, x_{j}}^{\epsilon}\left(x_{0}+P^{\epsilon} y, t_{0}\right)+C \lambda\left|D h^{\epsilon}(y)\right|^{2} .
\end{aligned}
$$

If $\lambda$ is chosen sufficiently small, we deduce

$$
\left|D h^{\epsilon}(y)\right| \leqslant 2\left|\nabla u_{t}^{\epsilon}\left(x_{0}+P^{\epsilon} y, t_{0}\right)\right| \leqslant C \epsilon^{-2}
$$

by (4.21). This estimate together with (1.5) yields

$$
\left\|\zeta u_{t}^{\epsilon}\left(x_{0}+P^{\epsilon} ., t_{0}\right)\right\|_{C^{0, \beta}\left(\bar{B}_{1}\right)} \leqslant C\left(1+R^{-\beta}+\epsilon^{-2} R^{1-\beta}\right) .
$$

If we substitute (4.33) as well as (4.32) and (4.30) into (4.25) we finally obtain

$$
\begin{aligned}
\left\|D^{2} z^{\epsilon}\right\|_{C^{0, \beta}\left(\bar{B}_{1}\right)} & \leqslant C \lambda\left\|D^{2} z^{\epsilon}\right\|_{C^{0, \beta}\left(\bar{B}_{1}\right)}+C\left(1+R^{-\beta}\right)+C \epsilon^{-2} R^{1-\beta} \\
& \leqslant C \lambda\left\|D^{2} z^{\epsilon}\right\|_{C^{0, \beta}\left(\bar{B}_{1}\right)}+C \epsilon^{-2 \beta}
\end{aligned}
$$

since $R=\lambda \epsilon^{2}$. Choosing $\lambda$ sufficiently small and observing that $\zeta \equiv 1$ on $B_{R}$ (4.24) follows and the lemma is proved. 


\section{REFERENCES}

1. Chen, Y.-G., Giga, Y., \& Goto, S. Uniqueness and existence of viscosity solutions of generalized mean curvature flow equations. J. Differ. Geom. 33, (1991) 749-786.

2. Chen, Y.-G., Giga, Y., Hitaka, Y. T., \& Honma, M. A stable difference scheme for computing motion of level surfaces by the mean curvature. In KIM, D. et al. (eds), Proceedings of the Global Analysis Research Center Symposium, Seoul, Korea, (1994), pp. 1-19.

3. Crandall, M. G., Ishit, H., \& LiOns, P. L. User's guide to viscosity solutions of second order partial differential equations. Bull. Am. Math. Soc. 27, (1992) 1-67.

4. Crandall, M. G. \& Lions, P. L. Convergent difference schemes for nonlinear parabolic equations and mean curvature motion. Numer. Math. 75, (1996) 17-41.

5. Deckelnick, K. \& DZIUK, G. Discrete anisotropic curvature flow of graphs. Preprint No 34, University of Freiburg (1996).

6. Deckelnick, K. \& Elliott, C. M. Finite element error bounds for a curve shrinking with prescribed normal contact to a fixed boundary. IMA J. Numer. Anal. 18, (1998) 635-654.

7. DzIUK, G. Convergence of a semi-discrete scheme for the curve shortening flow. Math. Models Methods Appl. Sci. 4, (1994) 589-606.

8. ECKER, K. \& HUISKen, G. Mean curvature evolution of entire graphs. Ann. Math. 130, (1989) 453-471.

9. Elliott, C. M. Approximation of curvature dependent interface motion. In DuFf, I. S. et al. (eds), The State of the Art in Numerical Analysis. 1996 Clarendon Press, Oxford. Inst. Math. Appl. Conf. Ser., New Ser. 63, (1997) 407-440.

10. Evans, L. C. \& Spruck, J. Motion of level sets by mean curvature I. J. Differ. Geom. 33, (1991) 636-681.

11. Evans, L. C. \& Spruck, J. Motion of level sets by mean curvature IV. J. Geom. Anal. 5, (1995) 77-114.

12. FRIED, M. Berechnung des Krümmungsflusses von Niveauflächen. Diploma thesis, University of Freiburg (1993).

13. Gilbarg, D. \& Trudinger, N. S. Elliptic Partial Differential Equations of Second Order. SpringerVerlag, Berlin (1983).

14. GIRÃO, P. Convergence of a crystalline algorithm for the motion of a simple closed convex curve by weighted curvature. SIAM J. Numer. Anal. 32, (1995) 886-899.

15. Huisken, G. Flow by mean curvature of convex surfaces into spheres. J. Differ. Geom. 20, (1984) 237266.

16. Huisken, G. Asymptotic behavior for singularities of the mean curvature flow. J. Differ. Geom. 31, (1990) 285-299.

17. Nochetto, R. H. \& Verdi, C. Convergence past singularities for a fully discrete approximation of curvature driven interfaces. SIAM J. Numer. Anal. 34, (1997) 490-512.

18. Osher, S. \& Sethian, J. A. Fronts propagating with curvature dependent speed: algorithms based on Hamilton-Jacobi formulations. J. Comput. Phys. 79, (1988) 12-49.

19. Sethian, J. A. Level set methods. Cambridge Monographs on Applied and Computational Mathematics 3. Cambridge University Press, Cambridge (1996).

20. Walkington, N. J. Algorithms for computing motion by mean curvature. SIAM J. Numer. Anal. 33, (1996) 2215-2238. 